\title{
Modified form of the Kompaneets equation
}

\section{Possible application to the distortion of high-temperature blackbody and CMB spectra ${ }^{\star}$}

\author{
Xurong Chen ${ }^{1}$, Xu Zhang ${ }^{1,2}$, Haixiang Gao ${ }^{3}$, Chengdong Han ${ }^{1,2}$, and Dangbo Liu $^{4,5}$ \\ ${ }^{1}$ Institute of Modern Physics, Chinese Academy of Sciences, Lanzhou 730000, PR China \\ e-mail: xchen@impcas.ac.cn \\ 2 University of Chinese Academy of Sciences, Beijing 100049, PR China \\ 3 Zhiyuan College, Shanghai Jiao Tong University, 800 Dongchuan Road, 200240 Shanghai, PR China \\ 4 Department of Astronomy, School of Physics and Astronomy, Shanghai Jiao Tong University, 800 Dongchuan Road, \\ 200240 Shanghai, PR China \\ e-mail: dbliu@sjtu.edu.cn \\ 5 Shanghai Key Laboratory for Particle Physics and Cosmology, Shanghai Jiao Tong University, 800 Dongchuan Road, \\ 200240 Shanghai, PR China
}

Received 15 August 2019 / Accepted 26 May 2020

\begin{abstract}
Comptonization is a very important phenomenon in astrophysics. The Kompaneets equation describes the Comptonization process in up-Comptonization $\left(h \bar{v} \ll k T_{\mathrm{e}}\right)$, while it fails in describing the down-Comptonization $\left(h \bar{v} \gg k T_{\mathrm{e}}\right)$, which is the most important radiative transfer process in hard X-ray and $\gamma$-ray astronomy. In this study we extend the Kompaneets equation to a new modified equation which works in a more general Comptonization process, including up- and down-Comptonization, suitable for the cases $h \bar{v} \ll k T_{\mathrm{e}}, h \bar{v} \gg k T_{\mathrm{e}}$, and $h \bar{v} \sim k T_{\mathrm{e}}$. Numerical solutions for the evolution behavior of Gaussian emission lines show excellent agreement between the classical equations and our new equation for up-Comptonization, while the big differences are displayed for down-Comptonization. Based on this extended equation, the modified Sunyaev-Zel'dovich effect is investigated. Instead, some typical calculated results for up- and down-Comptonization in X-ray astronomy are compared between the new equation and the Kompaneets equation. The potential applications of the extended equation in astrophysics are also highly emphasized for further study.
\end{abstract}

Key words. scattering - radiative transfer - methods: analytical - X-rays: ISM - gamma rays: ISM

\section{Introduction}

Radiative transfer for photons passing through a plasma (i.e., photon-electron scattering) is an important topic in both astrophysics and radiation physics. The transfer process, known as Comptonization, significantly affects the properties of emergent radiation from plasmas, for example the spectral shape of the continuum, the profile and centroid energy of an emission or absorption line, the intensity ratio of different lines, and the polarization (Fabian et al. 1995; Misra \& Kembhavi 1998; Titarchuk 1994). The detailed consideration of the problem of energy transfer between the radiation and the medium is as important as that of the basic radiation mechanism, particularly in the X-ray band. With the rapid development of X-ray astronomy in recent years, it seems necessary to have an effective theory that describes the radiative transfer process (Kompaneets 1957; Illarionov et al. 1979; Fabian et al. 1995; Sunyaev \& Titarchuk 1985; Titarchuk 1994; Hua et al. 1997; Kazanas et al. 1997; Misra \& Kembhavi 1998; Zhang et al. 2000; Liu et al. 2004; Felten \& Rees 1972; Miyamoto 1978). When high-energy photons go through the

* Research supported by the Strategic Priority Research Program of Chinese Academy of Sciences, Grant No. XDB34030301, by the National Science Foundation of China (Grant Nos. U1631101, 11665022, 11233006), and by the Shanghai Science and Technology Commission (Grant No. 16ZR1417200). media surrounding them, these media may be ionized into plasma. We note the specialty of the radiative transfer in X-ray band: for an almost fully ionized plasma at high temperature, which often occurs in high-energy astrophysics, the dominant mechanism of energy exchange between radiation and plasma is nonelastic photon-electron scattering. This special transfer mechanism in Xray astronomy is known as Comptonization. Comptonization is also of vital importance in astro-particle physics.

Although the change of the photon wavelength (or energy) in each individual collision between a photon and an electron is very small, the integrated variation in photon energy in multiple Compton scatterings in actual astronomical circumstances may be significant, particularly for the hard X-ray and $\gamma$-ray photons. The fractional change of the photon wavelength or frequency in each collision is given by the well-known formula (if the electron is approximately motionless before collision)

$$
\frac{\Delta \lambda}{\lambda}=\frac{\lambda^{\prime}-\lambda_{0}}{\lambda_{0}}=\frac{2 \lambda_{\mathrm{C}}}{\lambda_{0}} \sin ^{2} \frac{\theta}{2} \approx \frac{\lambda_{\mathrm{C}}}{\lambda_{0}}
$$

where $\lambda_{\mathrm{C}} \equiv h / m_{\mathrm{e}} c=0.024 \AA$ is the Compton wavelength and $\theta$ is the scattering angle. It is seen from Eq. (1) that the fractional change $\frac{\Delta \lambda}{\lambda_{0}}$ depends on the initial wavelength $\lambda_{0}$. The shorter the initial wavelength $\lambda_{0}$ is, the larger the change $\frac{\Delta \lambda}{\lambda_{0}}$ will be. For example, $\frac{\Delta \lambda}{\lambda_{0}} \approx 10^{-6}$ for $\lambda_{0}=5000 \AA$ in the optical band, but 
$\frac{\Delta \lambda}{\lambda_{0}} \approx 10^{-2}$ if $\lambda_{0} \simeq 0.5 \AA$ ( $h v \sim 20 \mathrm{keV}$, in the $\mathrm{X}$-ray band). For a very hard $\gamma$-ray photon with energy $\sim 100 \mathrm{keV}, \frac{\Delta \lambda}{\lambda_{0}} \approx 10^{-1}$. This is why Comptonization is particularly important in hard X-ray and $\gamma$-ray astronomy.

Generally, we use the Kompaneets equation to describe Comptonization,

$\left(\frac{\partial n}{\partial t}\right)_{c}=\frac{k T_{\mathrm{e}}}{m_{\mathrm{e}} c^{2}} N_{\mathrm{e}} \sigma_{\mathrm{T}} c \frac{1}{x^{2}} \frac{\partial}{\partial x}\left\{x^{4}\left[\frac{\partial n}{\partial x}+n(n+1)\right]\right\}$,

where $x \equiv h v / k T_{\mathrm{e}}$ is the dimensionless photon energy; $k T_{\mathrm{e}}$ is the electron temperature; $N_{\mathrm{e}}$ is the number density of the scattering electron gas; $\sigma_{\mathrm{T}}$ is the Thomson cross section; and $n(x, t) \equiv$ $n(v, t)$ is the frequency distribution function of the photon gas. The Kompaneets equation describes the Comptonization scattering of low-energy photons of frequency $v$ on a dilute distribution of nonrelativistic electrons when all photons and electrons are distributed isotropically in their momenta. The Kompaneets equation is applicable when the photon energy $h v \ll k T_{\mathrm{e}} \ll$ $m_{\mathrm{e}} c^{2}$. However, it fails in describing the Comptonization of highenergy photons passing through electron plasma, which is the most important radiative transfer process in hard X-ray and $\gamma$ ray astronomy.

Based on the Fokker-Plank equation, Ross et al. (1978) obtained the Ross-McCray equation to describe Compton scattering:

$\left(\frac{\partial n}{\partial t}\right)_{c}=\frac{k T_{\mathrm{e}}}{m_{\mathrm{e}} c^{2}} N_{\mathrm{e}} \sigma_{\mathrm{T}} c \frac{1}{x^{2}} \frac{\partial}{\partial x}\left\{x^{4}\left[n+\left(1+\frac{7}{10} \frac{k T_{\mathrm{e}}}{m_{\mathrm{e}} c^{2}} x^{2}\right) \frac{\partial n}{\partial x}\right]\right\}$.

Strictly, for the diffusion equation, when the photon gas reaches a thermal equilibrium with the electrons, $\frac{\partial n}{\partial t}=0$ should be satisfied. While inserting the Planck distribution function $n(x)=$ $\left(e^{x}-1\right)^{-1}$ into Eq. (3), $\frac{\partial n}{\partial t} \neq 0$. This means that the Ross-McCray equation does not conserve the number of photons.

Liu et al. (2004) extended the Kompaneets equation, with the goal of describing a more general Compton scattering process:

$$
\begin{aligned}
\left(\frac{\partial n}{\partial t}\right)_{c}= & \frac{k T_{\mathrm{e}}}{m_{\mathrm{e}} c^{2}} N_{\mathrm{e}} \sigma_{\mathrm{T}} c \frac{1}{x^{2}} \\
& \times \frac{\partial}{\partial x}\left\{x^{4}\left(1+\frac{7}{10} \frac{k T_{\mathrm{e}}}{m_{\mathrm{e}} c^{2}} x^{2}\right)\left[\frac{\partial n}{\partial x}+n(n+1)\right]\right\} .
\end{aligned}
$$

Kompaneets \& Eksper (1956), Kompaneets (1957) assumed that $\Delta v$ is also a small quantity when $h v \gg k T_{\mathrm{e}}$. Then he used $\Delta v$ to expand the distribution function, but this condition is not always satisfied. The change in photon energy is (Kompaneets 1957)

$$
h \Delta v=-\frac{h v c \boldsymbol{p} \cdot\left(\boldsymbol{n}-\boldsymbol{n}^{\prime}\right)+(h v)^{2}\left(1-\boldsymbol{n} \cdot \boldsymbol{n}^{\prime}\right)}{m_{\mathrm{e}} c^{2}\left[1+\left(h v / m_{\mathrm{e}} c^{2}\right)\left(1-\boldsymbol{n} \cdot \boldsymbol{n}^{\prime}\right)-\boldsymbol{p} \cdot \boldsymbol{n}^{\prime} /\left(m_{\mathrm{e}} c\right)\right]} .
$$

The momentum of thermal electrons is $p \sim m_{\mathrm{e}} v \sim m_{\mathrm{e}} \sqrt{k T_{\mathrm{e}} / m_{\mathrm{e}}}$. So in Eq. (4) the ratio of the second term in the numerator to the first term is $\sqrt{\left(h v / m_{\mathrm{e}} c^{2}\right)\left(h v / k T_{\mathrm{e}}\right)}$. When $h v$ is large enough, as Liu et al. (2004) argued, the second term in the numerator cannot be neglected. Hence in this paper we keep the second term. It is the first difference between our derivation and Kompaneets'. The second difference is that we re-derive the Kompaneets equation by expanding the distribution function with respect to the change in the electron's momentum $\Delta p$. We see that $\Delta p$ expansion is more reasonable than $\Delta v$ in both cases: $h v \gg k T_{\mathrm{e}}$ and $h v \ll k T_{\mathrm{e}}$.

This paper is organized as follows. In Sect. 2 we follow the Kompaneets method to improve the derivation of the diffuse equation for describing the Comptonization process of photon gas, and obtain a new modified form of the Kompaneets equation in a novel way. Then in Sect. 3 we compare our new equation with the Kompaneets equation by calculating the spectral evolution behavior of the Gaussian emission lines. In Sect. 4, based on the newly obtained equation and the Kompaneets equation, the evolution behavior of the blackbody spectra are displayed under some typical circumstances for high-energy astrophysics, and the Sunyaev-Zel'dovich effect is also investigated by involving our new equation. Finally, our conclusions and discussions are given in Sect. 5, in which the potential applications of the extended equation in astrophysics are highly emphasized. Additional Comptonization figures of the spectrum under various conditions can be found in Appendix A.

\section{New approach to modify the Kompaneets equation}

According to the Kompaneets method (Kompaneets \& Eksper 1956; Kompaneets 1957), we treat the radiation as a closed system that consists of a photon gas and an electron gas. Although the system cannot be described by a characteristic temperature before the thermal equilibrium is established, the electron gas itself is already in thermal equilibrium as the interaction between electrons is the Coulomb long-range force. For the tenuous plasma, the Fermi distribution of electron gas approximately equals the Boltzmann distribution:

$f(p)=f_{0} \exp \left[-\frac{1}{k T_{\mathrm{e}}} \frac{p^{2}}{2 m_{\mathrm{e}}}\right]$.

Because the photon is a boson, it does not affect the electron gas distribution, but it is capable of absorption and radiation of energy of all frequencies. Over a sufficiently long time, the absorption and emission of the photon gas caused by the scattering of electrons and photons will lead to thermal equilibrium.

In the electron and photon collision process such that $(\boldsymbol{p}, v, \boldsymbol{n}) \rightarrow\left(\boldsymbol{p}^{\prime}, v^{\prime}, \boldsymbol{n}^{\prime}\right)$, there will be a decrease in the photon number $n(v, t)$, corresponding to the fact that the number of electrons in the interval $\boldsymbol{p}-\boldsymbol{p}+\mathrm{d}^{3} \boldsymbol{p}$ is $N_{\mathrm{e}} f(\boldsymbol{p}) \mathrm{d}^{3} \boldsymbol{p}$. As the photon is the boson, the total transition number in unit volume is

$n\left(1+n^{\prime}\right) N_{\mathrm{e}} f(\boldsymbol{p}) \mathrm{d}^{3} \boldsymbol{p} \mathrm{d} \omega$.

The inverse process $\left(\boldsymbol{p}^{\prime}, v^{\prime}, \boldsymbol{n}^{\prime}\right) \rightarrow(\boldsymbol{p}, v, \boldsymbol{n})$ leads to an increase in the photon number $n(v, t)$, the total transition number per unit volume is

$n^{\prime}(1+n) N_{\mathrm{e}} f\left(\boldsymbol{p}^{\prime}\right) \mathrm{d}^{3} \boldsymbol{p}^{\prime} \mathrm{d} \omega$

where $n=n(v, t)$ and $n^{\prime}=n\left(v^{\prime}, t\right)$ are the photon numbers before and after the collision, respectively. Here $\boldsymbol{p}$ and $\boldsymbol{p}^{\prime}$ are the electron momentum before and after the collision. The transition probability $\mathrm{d} \omega$ is the same as in the collisions process $(\boldsymbol{p}, v, \boldsymbol{n}) \rightarrow$ $\left(\boldsymbol{p}^{\prime}, v^{\prime}, \boldsymbol{n}^{\prime}\right)$ and the inverse process $\left(\boldsymbol{p}^{\prime}, v^{\prime}, \boldsymbol{n}^{\prime}\right) \rightarrow(\boldsymbol{p}, v, \boldsymbol{n})$ because in the nonrelativistic limit the Compton differential scattering cross section can be approximately expressed by the Thomson cross section:

$\mathrm{d} W=c \mathrm{~d} \sigma_{\mathrm{T}}=c \frac{r_{0}^{2}}{2}\left(1+\cos ^{2} \theta\right) 2 \pi \sin \theta \mathrm{d} \theta$.

The Klein-Nishina cross section $\mathrm{d} \sigma_{\mathrm{T}} / \mathrm{d} \theta$ has the same value for the scattering angle $\theta$ and for $\pi-\theta$. Therefore, the change 
in the distribution function $n(v, t)$ that results from photon and electron scattering is

$\left(\frac{\partial n}{\partial t}\right)_{c}=-N_{\mathrm{e}} \int \mathrm{d}^{3} \boldsymbol{p} \int\left[n\left(1+n^{\prime}\right) f(\boldsymbol{p})-n^{\prime}(1+n) f\left(\boldsymbol{p}^{\prime}\right)\right] \mathrm{d} W$.

The laws of energy and momentum conservation in the nonrelativistic approximation are written in the form

$$
\begin{aligned}
& \frac{h v}{c} \boldsymbol{n}+\boldsymbol{p}=\frac{h v^{\prime}}{c} \boldsymbol{n}^{\prime}+\boldsymbol{p}^{\prime} \\
& h v+\frac{p^{2}}{2 m_{\mathrm{e}}}=h v^{\prime}+\frac{p^{\prime 2}}{2 m_{\mathrm{e}}} .
\end{aligned}
$$

Here $\boldsymbol{n}$ and $\boldsymbol{n}^{\prime}$ are the direction of photon before and after the collision, respectively. The expressions $\Delta v=v^{\prime}-v$ and $\Delta p=$ $\boldsymbol{p}^{\prime}-\boldsymbol{p}$ can be obtained from Eq. (11). Keeping only the firstorder terms in $\Delta v$, we obtain Eq. (5). In hard X-ray and $\gamma$-ray astronomy in most cases we apply $h v \ll m_{\mathrm{e}} c^{2}$ and $k T_{\mathrm{e}} \ll m_{\mathrm{e}} c^{2}$ to get the expression of $\Delta v$ :

$h \Delta v=-\frac{h v c}{m_{\mathrm{e}} c^{2}} \boldsymbol{p} \cdot\left(\boldsymbol{n}-\boldsymbol{n}^{\prime}\right)-\frac{(h v)^{2}}{m_{\mathrm{e}} c^{2}}\left(1-\boldsymbol{n} \cdot \boldsymbol{n}^{\prime}\right)$.

We can also obtain $\Delta v$ and $|\Delta p|$ from two equations in Eq. (11):

$h \Delta v=-\frac{1}{2 m_{\mathrm{e}}}\left[2|\boldsymbol{p}||\Delta \boldsymbol{p}| \cos \gamma+|\Delta \boldsymbol{p}|^{2}\right]$,

$|\Delta \boldsymbol{p}|^{2}=\left(\frac{h v}{c}\right)^{2}\left(\boldsymbol{n}-\boldsymbol{n}^{\prime}\right)^{2}-2\left(\boldsymbol{n}-\boldsymbol{n}^{\prime}\right) \cdot \boldsymbol{n}^{\prime}\left(\frac{h v}{c}\right)\left(\frac{h \Delta v}{c}\right)+\left(\frac{h \Delta v}{c}\right)^{2}$,

where $\gamma$ is the angle between $p$ and $\Delta p$.

Next, let us compare $\Delta p$ and $\Delta v$. The Compton scattering and the inverse Compton scattering consist of the whole scattering process of the photon and electron. When $\cos \gamma>0$, it is obvious that $\left|\frac{1}{2 m_{\mathrm{e}}} 2 p \Delta p \cos \gamma\right|<|h \Delta v|$. So we just need to consider the case $\cos \gamma<0$.

In inverse Compton scattering, a low-energy photon collides with a high-energy electron $\left(k T_{\mathrm{e}}>h v\right), h \Delta v>0$; In Compton scattering we have $h \Delta v<0$. Using Eqs. (13) and (14), we obtain

$$
\begin{aligned}
-\frac{1}{2 m_{\mathrm{e}}} 2|\boldsymbol{p}||\Delta \boldsymbol{p}| \cos \gamma= & -\left[\frac{h v c}{m_{\mathrm{e}} c^{2}} \boldsymbol{p} \cdot\left(\boldsymbol{n}-\boldsymbol{n}^{\prime}\right)\right] \\
& +\left(1-\boldsymbol{n} \cdot \boldsymbol{n}^{\prime}\right)\left(\frac{h v}{m_{\mathrm{e}} c^{2}}\right) h \Delta v+\frac{(h \Delta v)^{2}}{2 m_{\mathrm{e}} c^{2}} .
\end{aligned}
$$

The first term in Eq. (15) was used to derive the classical Kompaneets equation for the up-Comptonization process, which is applied to nonrelativistic astrophysics problems. In our approach, we keep the second term. The last term is a small quantity compared to other terms, and therefore can be neglected. The second term $\left(1-\boldsymbol{n} \cdot \boldsymbol{n}^{\prime}\right)\left(\frac{h v}{m_{\mathrm{e}} c^{2}}\right) h \Delta v$ is less than zero, and the first term is greater than zero, thus we obtain

$\left|-\frac{1}{2 m_{\mathrm{e}}} 2\right| \boldsymbol{p}|| \Delta \boldsymbol{p}|\cos \gamma|<\mid-\left[\frac{h v c}{m_{\mathrm{e}} c^{2}} \boldsymbol{p} \cdot\left(\boldsymbol{n}-\boldsymbol{n}^{\prime}\right)\right]$.

For the case, $h v \gg k T_{\mathrm{e}}$,

$\left|-\left[\frac{h v c}{m_{\mathrm{e}} c^{2}} \boldsymbol{p} \cdot\left(\boldsymbol{n}-\boldsymbol{n}^{\prime}\right)\right]\right| \ll \frac{(h v)^{2}}{m_{\mathrm{e}} c^{2}}\left(1-\boldsymbol{n} \cdot \boldsymbol{n}^{\prime}\right)$.
The first term in Eq. (12) can be neglected, we have

$h \Delta v \approx-\frac{(h v)^{2}}{m_{\mathrm{e}} c^{2}}\left(1-\boldsymbol{n} \cdot \boldsymbol{n}^{\prime}\right)$.

Therefore, combining Eqs. (16) and (18), we have

$\left|-\frac{1}{m_{\mathrm{e}}}\right| \boldsymbol{p}|| \Delta \boldsymbol{p}|\cos \gamma| \ll|h \Delta v|$.

Similarly, if $h v \ll k T_{\mathrm{e}}$, then

$\left|-\left[\frac{h v c}{m_{\mathrm{e}} c^{2}} \boldsymbol{p} \cdot\left(\boldsymbol{n}-\boldsymbol{n}^{\prime}\right)\right]\right| \approx|h \Delta v|$.

Thus, we have

$\left|-\frac{1}{m_{\mathrm{e}}}\right| \boldsymbol{p}|| \Delta \boldsymbol{p}|\cos \gamma|<|h \Delta v|$.

We have proved that $\left|-\frac{1}{m_{\mathrm{e}}}\right| \boldsymbol{p}|| \Delta \boldsymbol{p}|\cos \gamma|$ is smaller than $h \Delta v$ in both of the cases $h v \gg k T_{\mathrm{e}}$ and $h v \ll k T_{\mathrm{e}}$. We believe that it should be more precise to expand the distribution function in $\Delta p$ than in $\Delta v$. For convenience, we use $\Delta p$ to denote $|\Delta p|$. We expand $n^{\prime}=n\left(v^{\prime}, t\right)$ and $f\left(\boldsymbol{p}^{\prime}\right)$ in terms of $\Delta p$ to the second order by applying Eq. (13):

$$
\begin{aligned}
n^{\prime}=n & +\frac{\partial n}{\partial x}\left[-\frac{1}{k T_{\mathrm{e}}} \frac{1}{2 m_{\mathrm{e}}} 2 p \cos \gamma \Delta p-\frac{1}{k T_{\mathrm{e}}} \frac{1}{2 m_{\mathrm{e}}}(\Delta p)^{2}\right] \\
+ & \frac{1}{2} \frac{\partial^{2} n}{\partial x^{2}}\left[-\frac{1}{k T_{\mathrm{e}}} \frac{1}{2 m_{\mathrm{e}}} 2 p \cos \gamma \Delta p\right]^{2} \\
f\left(p^{\prime}\right)= & f_{0} \exp \left[-\frac{1}{k T_{\mathrm{e}}} \frac{1}{2 m_{\mathrm{e}}}(\boldsymbol{p}+\Delta \boldsymbol{p})^{2}\right] \\
= & f(p)+f(p)\left[-\frac{1}{k T_{\mathrm{e}}} \frac{1}{2 m_{\mathrm{e}}} 2 p \cos \gamma \Delta p-\frac{1}{k T_{\mathrm{e}}} \frac{1}{2 m_{\mathrm{e}}}(\Delta p)^{2}\right] \\
& +\frac{1}{2} f(p)\left[-\frac{1}{k T_{\mathrm{e}}} \frac{1}{2 m_{\mathrm{e}}} 2 p \cos \gamma \Delta p\right]^{2} .
\end{aligned}
$$

The first order is the same as expanding $n^{\prime}=n\left(v^{\prime}, t\right)$ and $f\left(\boldsymbol{p}^{\prime}\right)$ in terms of $\Delta v$, while there is a difference for the second order. This difference will result in new correctness.

Inserting Eqs. (22) and (23) into Eq. (10), and neglecting $\Delta p$ of order higher than 2 , we obtain

$$
\begin{aligned}
\left(\frac{\partial n}{\partial t}\right)_{c}= & N_{\mathrm{e}} \\
{[} & \left.\frac{\partial n}{\partial x}+n(n+1)\right] \int \mathrm{d}^{3} \boldsymbol{p} \int \mathrm{d} W \\
& \times f(p)\left\{\left[-\frac{1}{k T_{\mathrm{e}}} \frac{1}{2 m_{\mathrm{e}}} 2 p \cos \gamma\right] \Delta p-\frac{1}{k T_{\mathrm{e}}} \frac{1}{2 m_{\mathrm{e}}}(\Delta p)^{2}\right\} \\
+ & \frac{N_{\mathrm{e}}}{2}\left[\frac{\partial^{2} n}{\partial x^{2}}+2(n+1) \frac{\partial n}{\partial x}+n(n+1)\right] \int \mathrm{d}^{3} \boldsymbol{p} \int \mathrm{d} W \\
& \times f(p)\left\{\left[-\frac{1}{k T_{\mathrm{e}}} \frac{1}{2 m_{\mathrm{e}}} 2 p \cos \gamma\right]^{2}(\Delta p)^{2}\right\} .
\end{aligned}
$$

Next, following the Kompaneets method, we first calculate the second integral of Eq. (24). The other is determined from the condition that the equation ought to guarantee conservation of the total number of photons in the scattering process. By applying Eq. (13), let

$$
\begin{aligned}
I & =\int \mathrm{d}^{3} \boldsymbol{p} \int \mathrm{d} W f(p)\left\{\left[-\frac{1}{2 m_{\mathrm{e}}} 2 p \cos \gamma\right]^{2}(\Delta p)^{2}\right\} \\
& =\int \mathrm{d}^{3} \boldsymbol{p} \int \mathrm{d} W f(p)\left[h \Delta v+\frac{1}{2 m_{\mathrm{e}}}|\Delta \boldsymbol{p}|^{2}\right]^{2} .
\end{aligned}
$$


Inserting Eq. (14) into the above Eq. (25), we obtain

$$
\begin{aligned}
I= & \int \mathrm{d}^{3} \boldsymbol{p} \int \mathrm{d} W f(p)\left\{(h \Delta v)^{2}+\frac{1}{4 m_{\mathrm{e}}^{2}}\left[\left(\frac{h v}{c}\right)^{2}\left(\boldsymbol{n}-\boldsymbol{n}^{\prime}\right)^{2}\right.\right. \\
& \left.-2 \frac{h v}{c} \frac{h \Delta v}{c}\left(\boldsymbol{n}-\boldsymbol{n}^{\prime}\right) \cdot \boldsymbol{n}^{\prime}+\left(\frac{h \Delta v}{c}\right)^{2}\right]^{2} \\
+ & \frac{1}{m_{\mathrm{e}}}(h \Delta v)\left[\left(\frac{h v}{c}\right)^{2}\left(\boldsymbol{n}-\boldsymbol{n}^{\prime}\right)^{2}\right. \\
& \left.\left.-2 \frac{h v}{c} \frac{h \Delta v}{c}\left(\boldsymbol{n}-\boldsymbol{n}^{\prime}\right) \cdot \boldsymbol{n}^{\prime}+\left(\frac{h \Delta v}{c}\right)^{2}\right]\right\}
\end{aligned}
$$

Let

$$
\begin{aligned}
I_{1}= & \int \mathrm{d}^{3} \boldsymbol{p} \int \mathrm{d} W f(p)(h \Delta v)^{2} \\
I_{2}= & \int \mathrm{d}^{3} \boldsymbol{p} \int \mathrm{d} W f(p) \frac{1}{4 m_{\mathrm{e}}^{2}} \\
& \times\left[\left(\frac{h v}{c}\right)^{2}\left(\boldsymbol{n}-\boldsymbol{n}^{\prime}\right)^{2}-2 \frac{h v}{c} \frac{h \Delta v}{c}\left(\boldsymbol{n}-\boldsymbol{n}^{\prime}\right) \cdot \boldsymbol{n}^{\prime}+\left(\frac{h \Delta v}{c}\right)^{2}\right]^{2}, \\
I_{3}= & \int \mathrm{d}^{3} \boldsymbol{p} \int \mathrm{d} W f(p) \frac{1}{m_{\mathrm{e}}}(h \Delta v) \\
& \times\left[\left(\frac{h v}{c}\right)^{2}\left(\boldsymbol{n}-\boldsymbol{n}^{\prime}\right)^{2}-2 \frac{h v}{c} \frac{h \Delta v}{c}\left(\boldsymbol{n}-\boldsymbol{n}^{\prime}\right) \cdot \boldsymbol{n}^{\prime}+\left(\frac{h \Delta v}{c}\right)^{2}\right] .
\end{aligned}
$$

Then, completing the calculation of the above integrals, we can obtain

$I_{1}=\frac{2 k T_{\mathrm{e}}}{m_{\mathrm{e}} c^{2}}(h v)^{2} \sigma_{\mathrm{T}} c+\frac{(h v)^{4}}{m_{\mathrm{e}}^{2} c^{4}} \frac{7}{5} \sigma_{\mathrm{T}} c$,

$I_{2}=\frac{(h v)^{4}}{m_{\mathrm{e}}^{2} c^{4}} \frac{7}{5} \sigma_{\mathrm{T}} c$,

$I_{3}=-\frac{(h v)^{4}}{m_{\mathrm{e}}^{2} c^{4}} \frac{14}{5} \sigma_{\mathrm{T}} c+\frac{28}{5} \frac{k T_{\mathrm{e}}}{\left(m_{\mathrm{e}} c^{2}\right)^{2}}(h v)^{3} \sigma_{\mathrm{T}} c$.

Hence, the expression of the integral $I$ can be given as

$I \approx \frac{2 k T_{\mathrm{e}}}{m_{\mathrm{e}} c^{2}}(h v)^{2} \sigma_{\mathrm{T}} c+\frac{28}{5} \frac{k T_{\mathrm{e}}}{\left(m_{\mathrm{e}} c^{2}\right)^{2}}(h v)^{3} \sigma_{\mathrm{T}} c$.

Equation (24) should satisfy the conservation principle in the frequency space; therefore, using spherical coordinates it can be expressed as

$\left(\frac{\partial n}{\partial t}\right)_{c}=-\nabla \cdot \boldsymbol{j}=-x^{-2} \frac{\partial\left(x^{2} j\right)}{\partial x}$,

where $\boldsymbol{j}$ is the flux of photons defined in the frequency space, and $x \equiv h v /\left(k T_{\mathrm{e}}\right)$. Thus, Eq. (28) can be rewritten as

$\left(\frac{\partial n}{\partial t}\right)_{c}=-\frac{2}{x} j-\frac{\partial j}{\partial x}$.

Equation (24) is of the second order relative to $x$, and dependent on the second derivative $\frac{\partial^{2} n}{\partial x^{2}}$ linearly, so that the current must contain the first derivative $\frac{\partial n}{\partial x}$. Instead, in the state of thermal equilibrium, the distribution function is Planckian, $n(x)=$ $\left(e^{x}-1\right)^{-1}$, and the flow vanishes. Thus, $\frac{\partial n}{\partial x}=-n(n+1)$. Therefore, the flux $j$ may be given in the form:

$j(x)=g(x)\left[\frac{\partial n}{\partial x}+n(n+1)\right]$.

Inserting Eq. (30) into Eq. (29), we obtain

$$
\begin{aligned}
\left(\frac{\partial n}{\partial t}\right)_{c}= & -g(x)\left[\frac{\partial^{2} n}{\partial x^{2}}+(2 n+1) \frac{\partial n}{\partial x}\right] \\
& -\left[\frac{\partial g}{\partial x}+\frac{2 g}{x}\right]\left[\frac{\partial n}{\partial x}+n(n+1)\right] .
\end{aligned}
$$

Equation (24) can be re-written as

$$
\begin{aligned}
\left(\frac{\partial n}{\partial t}\right)_{c}= & N_{\mathrm{e}} \frac{1}{k T_{\mathrm{e}}}\left[\frac{\partial n}{\partial x}+n(n+1)\right] H \\
& +\frac{N_{\mathrm{e}}}{2}\left(\frac{1}{k T_{\mathrm{e}}}\right)^{2}\left[\frac{\partial^{2} n}{\partial x^{2}}+2(n+1) \frac{\partial n}{\partial x}+n(n+1)\right] I,
\end{aligned}
$$

where $H \equiv H(x)$ represents the function of the dimensionless photon energy $x \equiv h v / k T_{\mathrm{e}}$, which is given below. Although it is difficult to calculate the function $H$ directly, it can be deduced simply from the integral value $I$ given by Eq. (27) from the consideration of conservation of total number of photons in the scattering process. Comparing Eq. (32) with Eq. (31) and noting that the coefficient of $\frac{\partial^{2} n}{\partial x^{2}}$ should be the same, the expression of $g(x)$ is found to be

$g(x)=-\frac{N_{\mathrm{e}}}{2}\left(\frac{1}{k T_{\mathrm{e}}}\right)^{2} I=-A x^{2}(1+B x)$,

where $A=\frac{k T_{\mathrm{e}}}{m_{\mathrm{e}} c^{2}} N_{\mathrm{e}} \sigma_{\mathrm{T}} c, B=\frac{14}{5} \frac{k T_{\mathrm{e}}}{m_{\mathrm{e}} c^{2}}$. Inserting Eq. (33) into Eq. (31) and comparing Eq. (31) with Eq. (32) again, we obtain

$H=\frac{k T_{\mathrm{e}}}{N_{\mathrm{e}}} A\left(4 x-x^{2}+5 B x^{2}-B x^{3}\right)$.

Therefore, the modified the Kompaneets equation under the condition $k T_{\mathrm{e}} \ll m_{\mathrm{e}} c^{2}$ and $h v \ll m_{\mathrm{e}} c^{2}$ can be obtained as follows:

$$
\begin{aligned}
\left(\frac{\partial n}{\partial t}\right)_{c}= & \frac{k T_{\mathrm{e}}}{m_{\mathrm{e}} c^{2}} N_{\mathrm{e}} \sigma_{\mathrm{T}} c \frac{1}{x^{2}} \\
& \times \frac{\partial}{\partial x}\left\{x^{4}\left(1+\frac{14}{5} \frac{k T_{\mathrm{e}}}{m_{\mathrm{e}} c^{2}} x\right)\left[\frac{\partial n}{\partial x}+n(n+1)\right]\right\} .
\end{aligned}
$$

Equation (35) is a significantly extended equation relative to the original Kompaneets equation (Eq. (2)) because the conditions $h v \ll m_{\mathrm{e}} c^{2}$ and $k T_{\mathrm{e}} \ll m_{\mathrm{e}} c^{2}$ are much looser than those of Eq. (2). The comparison between $h v$ and $k T_{\mathrm{e}}$ is no longer necessary, i.e., Eq. (35) can be applied for various cases: $h v \ll k T_{\mathrm{e}}$, $h v \simeq k T_{\mathrm{e}}$, and particularly for $h v \gg k T_{\mathrm{e}}$. It should be noted that we use $\Delta p$ rather than $\Delta v$ in the Taylor expansion to obtain the new equation. This is the main reason that our result is different from previous results. The new equation works with the photon energy $h v \ll m_{\mathrm{e}} c^{2}$ and the electron temperature $k T_{\mathrm{e}} \ll m_{\mathrm{e}} c^{2}$ to describe more general Comptonization, including both downand up-Comptonization. When the energy of photons $h v$ is low enough (i.e., $x \equiv h v / k T_{\mathrm{e}} \ll 1$ ), then the term $\frac{14}{5} \frac{k T_{\mathrm{e}}}{m_{\mathrm{e}} c^{2}} x$ in Eq. (35) is negligible, so we return to the classical Kompaneets equations. On the other hand, when the energy of photons $h v$ is very high, the term $\frac{14}{5} \frac{k T_{\mathrm{e}}}{m_{\mathrm{e}} c^{2}} x$ in Eq. (35) is non-negligible, especially for 
down-Comptonization $h \bar{v} \gg k T_{\mathrm{e}}$. Therefore, we note that our extended Kompaneets equation is better than the Kompaneets equation in describing the Comptonization of high-energy photons passing through "cold" electron plasma, which is of great significance in hard X-ray and $\gamma$-ray astronomy. We present the detailed numerical results to show the advantage of our new equation in the following.

Here we consider an ideal Comptonization process in infinite space. For real astrophysical situations, the medium is finite. To have a finite optical depth, we should limit evolution time. For example, if we choose the evolution time to be $0.01 \mathrm{~s}$, the optical depth can be evaluated using the given formula Eq. (36) in the following. For a bound medium, Titarchuk (1994) demonstrated that the emergent spectrum can be obtained as a convolution of the time-dependent energy diffusion solution. Titarchuk's theorem has been used for the solution of real astrophysical problems.

\section{Numerical calculations for the Comptonization of Gaussian emission lines: Comparison of the modified and original Kompaneets equations}

Using the diffusion equation Eq. (35), we can study the effect of Comptonization on the radiation (including X-ray, optical, infrared, and radio spectrum) emergent from the scattering medium. The effect of down-Comptonization, for which Eq. (2) is difficult to use, is especially emphasized. Given the initial condition, i.e., the initial spectrum, and the diffusion timescale, we can solve Eq. (35) numerically by using the three-point finite difference method (Liu et al. 2004). For simplicity, we assume that the X-ray source is stable with a time-independent spectrum $f(x)$. Hence, the initial spectrum of Eq. (35) can be expressed as $n(x, t=0)=f(x)$. The diffusion timescale $T$, which represents the average time before a trapped photon escapes from the surface of the scattering region of the electron gas, is determined by both the geometric size $L$ and the scattering depth $\tau_{\mathrm{s}}$ of the scattering region, where $L$ denotes either the radius of a spherical scattering cloud surrounding the central X-ray source or the thickness of a plane-parallel scattering slab dependent on the geometry of the problem. According to the random walk theory (Rybicki \& Lightman 1979), the average scattering number of a scattered photon before escaping is $N \approx \max \left(\tau_{\mathrm{s}}, \tau_{\mathrm{s}}^{2}\right) \approx \tau_{\mathrm{s}}^{2}$ for $\tau_{\mathrm{s}}>1$, where $\tau_{\mathrm{s}}=N_{\mathrm{e}} \sigma_{\mathrm{T}} L$ is the scattering depth. The average trapping $T=N \cdot(l / c)$, where $l=1 /\left(N_{\mathrm{e}} \sigma_{\mathrm{T}}\right)$ is the mean free path of Compton scattering. Therefore, we obtain

$T \simeq \tau_{\mathrm{s}}^{2} \frac{1}{N_{\mathrm{e}} \sigma_{\mathrm{T}} c}=\frac{\tau_{\mathrm{s}} L}{c} \quad$ for case $\tau_{\mathrm{s}}>1$.

In this paper we use Eq. (36) to estimate the diffusion timescale $T$ to get the final emergent spectrum.

The relationship between the average scattering number $N$ and the diffusion timescale $T$ is $N \simeq N_{\mathrm{e}} \sigma_{\mathrm{T}} c T$. When $N_{\mathrm{e}} \approx$ $2 \times 10^{16} \mathrm{~cm}^{-3}, T=1.0 \times 10^{-2} \mathrm{~s}$, it is obtained that $N \approx 4.0$. The multiplication term in Eq. (35) depends on the average scattering number $N$ only, which implies that we can also use $N$ instead of $T$ and $N_{\mathrm{e}}$ (or $T$ and $\tau_{\mathrm{s}}$ ) to determine the final emergent spectrum. For Eq. (36) we assumed a large scattering depth $\tau_{\mathrm{s}}>1$, optically thick, as otherwise the Comptonization process becomes unimportant. Combining Eqs. (35) and (36), we obtain the emergent Comptonization spectrum $n(x, T)$. It is known that the energy density of the radiation field is given by $u_{v} \mathrm{~d} v=\frac{8 \pi v^{2}}{c^{3}} n(v, t) h v \mathrm{~d} v \propto n x^{3}$; therefore, the spectral intensity is expressed by $I_{v}=\frac{c}{4 \pi} u_{v} \propto n x^{3}$, or $n(x, t) \propto x^{-3} I_{v}$.
Finally, the emergent spectral intensity $I$ could thus be obtained as $I(v, T) \equiv I(x, T) \propto x^{3} n(x, T)$.

It should be noted that this simplification neglects the spatial diffusion term, and the emergent spectrum obtained reflects the Comptonization result of the initial spectrum in an infinite homogeneous medium. For a finite medium with more complex geometry, Sunyaev \& Titarchuk (1980) have given a detailed discussion.

In this section, we adopt the initial spectrum of an emission line with Gaussian profile as an exercise of Eq. (35) to demonstrate the time-evolution behavior of emergent spectra from the scattering electron gas. The expression for the spectral intensity for an emission line with normal Gaussian profile is given as

$I(v) \sim \exp \left[-\frac{4 \ln 2}{(\Delta v)^{2}}\left(v-v_{0}\right)^{2}\right]$,

where $h v_{0}$ and $h \Delta v$ are the centroid energy and the FWHM of the Gaussian emission line, respectively.

Using the relation $I_{v}=\frac{2 h v^{3}}{c^{2}} n(v)$, the initial line profile of a stable source is given as

$n(x, 0)=f(x) \sim x^{-3} \exp \left[-\frac{4 \ln 2}{(\Delta x)^{2}}\left(x-x_{0}\right)^{2}\right]$,

where $x \equiv h v / k T_{\mathrm{e}}$ represents the dimensionless photon energy.

In order to reveal the discrepancy between Eqs. (2) and (35), we attempt to solve the two equations numerically under the same conditions. For simplicity and precision, we assume that the scattering medium is homogeneous in space with constant electron density $N_{\mathrm{e}}$. We fix $N_{\mathrm{e}} \approx 2 \times 10^{16} \mathrm{~cm}^{-3}$, which is the typical value for the plasma in the accretion disk around a compact star, for example a neutron star, a singular star, or a black hole with stellar mass (Treves et al. 1989; Shakura \& Sunyaev 1973). To enlarge the astronomical application of Eqs. (2) and (35), we abandon the assumption of a "tenuous radiation field", $n \ll 1$, adopted by Chen et al. (1994) and Deng et al. (1998), and retain the $n^{2}$ term in Eqs. (2) and (35). This means that our calculated results can be used to discuss very luminous high-energy objects with a strong radiation field.

By using Eqs. (35) and (38) in the following numerical calculations, the evolution behavior of photons with the spectrum of Gaussian line profile will be shown under different diffuse timescales $T$. Moreover, the calculated results of Kompaneets equation Eq. (2) are also given for comparison with our new obtained equation Eq. (35). The numerical solutions of our extended Eq. (35) and the Kompaneets equation Eq. (2) are shown in Figs. 1-3 (and Figs. A.1-A.5) for the cases $h v \ll$ $k T_{\mathrm{e}}, h v \sim k T_{\mathrm{e}}$, and $h v \gg k T_{\mathrm{e}}$, including both down- and up-Comptonization. In each figure, the black solid line represents the original line profile before the diffusion of photons (Comptonization); the blue dotted, the blue dash-dot-dotted, and the blue short-dotted curves respectively represent the emergent spectra calculated by Eq. (35) with different diffusion timescale $T=1.0 \times 10^{-2} \mathrm{~s}, T=5.0 \times 10^{-2} \mathrm{~s}$, and $T=1.0 \times 10^{-1} \mathrm{~s}$ under different given temperatures $k T_{\mathrm{e}}$ of electron gas. Instead, for comparison with the results obtained by Eq. (35), the emergent spectra calculated by Eq. (2) under the same above $k T_{\mathrm{e}}$ with time $T=1.0 \times 10^{-2} \mathrm{~s}, T=5.0 \times 10^{-2} \mathrm{~s}$, and $T=$ $1.0 \times 10^{-1} \mathrm{~s}$ are also exhibited in each figure by the red dashed, the red dash-dotted, and the red short-dashed curves, respectively. The given temperatures $k T_{\mathrm{e}}$ of electron gas are labeled in each figure. From Figs. 1-3 and A.1-A.5 it is easy to see the evolution behavior of the line profile with time $T$. When the low-energy line-photons pass through "hot" electron gas 
(i.e., $h v_{0}<k T_{\mathrm{e}}$; up-Comptonization), the evolution behavior of Gaussian line spectra with $h v_{0}=0.1 \mathrm{keV}, 1.0 \mathrm{keV}$, and $10 \mathrm{keV}$ is shown in Figs. 1, A.1, and A.2 respectively, whereas the evolution behavior for high-energy line-photons, passing through cold plasma (i.e., $h v_{0}>k T_{\mathrm{e}}$; down-Comptonization) is displayed in Figs. 2, 3, and A.3-A.5 for the line-photon energy $h v_{0}=1.0 \mathrm{keV}, 10 \mathrm{keV}, 50 \mathrm{keV}, 100 \mathrm{keV}$, and $200 \mathrm{keV}$, respectively.

As shown in Figs. 1, 2, and A.1-A.3, the line profiles in Compton evolution obtained by our extended equation Eq. (35) are nearly consistent with the ones obtained by the Kompaneets equation (Eq. (2)) when the energy of the line-photons is much lower than $m_{\mathrm{e}} c^{2}$, i.e., $h v_{0} \ll m_{\mathrm{e}} c^{2}$, including the cases $h v_{0}<k T_{\mathrm{e}}$ and $h v_{0}>k T_{\mathrm{e}}$ (see Figs. 1, A.1, and A.2; the differences between the corresponding curves are too small to be seen, except for Figs. A.2c and d), while the differences in the evolution behavior of the resultant line profiles for high-energy photons $h v_{0}>k T_{\mathrm{e}}$ between Eqs. (2) and (35) are more obvious with the increasing of energy of the photons, especially for much higher energy photons $h v_{0}$ greater than several tens $\mathrm{keV}$, even greater than several hundreds keV (see Figs. 3, A.4, and A.5). The big differences appear in Figs. 3, A.3, and A.4 for down-Comptonization $\left(h v_{0}>k T_{\mathrm{e}}\right)$ because the term $\frac{14}{5} \frac{k T_{\mathrm{e}}}{m_{\mathrm{e}} c^{2}} x$ in Eq. (35) cannot be negligible, which can bring a much more significant contribution to the evolution behavior of the high-energy photons. On the other hand, even for the up-Comptonization process, if the temperature $k T_{\mathrm{e}}$ of electron is high enough (e.g., $k T_{\mathrm{e}}>100 \mathrm{keV}$ ), more lower energy photons will be scattered to higher energy with the increase in the scattering time $T$, which also results in the difference of the evolution behavior between the extended Kompaneets equation and Kompaneets equation because the term $\frac{14}{5} \frac{k T_{\mathrm{e}}}{m_{\mathrm{e}} c^{2}} x$ in Eq. (35) becomes much more important for the Comptonization process of high-energy photons (e.g., see Figs. A.2c and $\mathrm{d}$ ).

From Figs. 1, A.1, and A.2 we see the main evolution behavior of the line profile in up-Comptonization, which are as follows: (i) The peak positions of the line profiles are almost shifted to higher energies. We call this the "Compton blueshift" of the line. The longer the diffusion time $T$, the higher the line blueshift will be; (ii) The line width increases with $T$, which shows a diffusion behavior in the frequency space; (iii) The line profile becomes asymmetrical, steeper on the low-energy side and flatter on the high-energy side. Instead, from Figs. 2, 3, and A.4-A.6 we can summarize the main evolution features of the line profile in down-Comptonization as follows: (i) The peak position of the line is shifted to lower energies. We call this the "Compton redshift" of the line. The longer the diffusion time $T$, the higher the line redshift will be; (ii) The evolution behavior of the line profile has two distinctive manners, which are determined by the balance of the photon gas and the electron gas. If the difference between the line-photon energy $h v_{0}$ and the temperature $k T_{\mathrm{e}}$ of electron gas is not very large, from Figs. 2a, 3a, and A.4a-A.6a we see that the line width increases with $T$, which shows a diffusion behavior in the frequency space; instead, if the difference between $h v_{0}$ and $k T_{\mathrm{e}}$ is much larger, it seems that the line profile is just collectively shifted to lower energy side and the line width becomes slightly narrower (see Figs. 2c, 3c, and A.4cA.6c); (iii) From Figs. 2a, 3a, and A.4a-A.6a we see that the line profiles also become asymmetrical, steeper on the low-energy side and flatter on the high-energy side, while for Figs. 2c, 3c, and A.4c-A.6c the line profiles are only slightly asymmetrical, steeper on both the low- and high-energy sides.
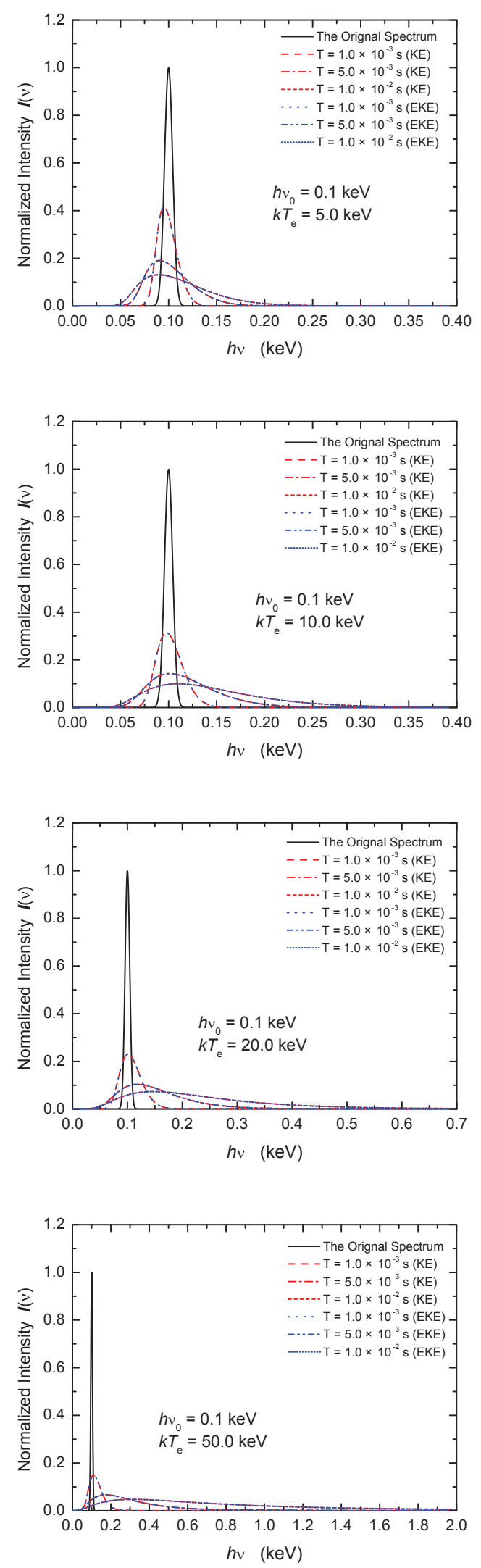

Fig. 1. Comparison of evolution behavior for the up-Comptonization of the Gaussian line with $h v_{0}=0.1 \mathrm{keV}$ between the classical Kompaneets equation and our extended equation. The evolution times $T, h v_{0}$, and $k T_{\mathrm{e}}$ are labeled in the figures. The blue and red lines represent the calculated results of the extended equation and the Kompaneets equation, respectively. 

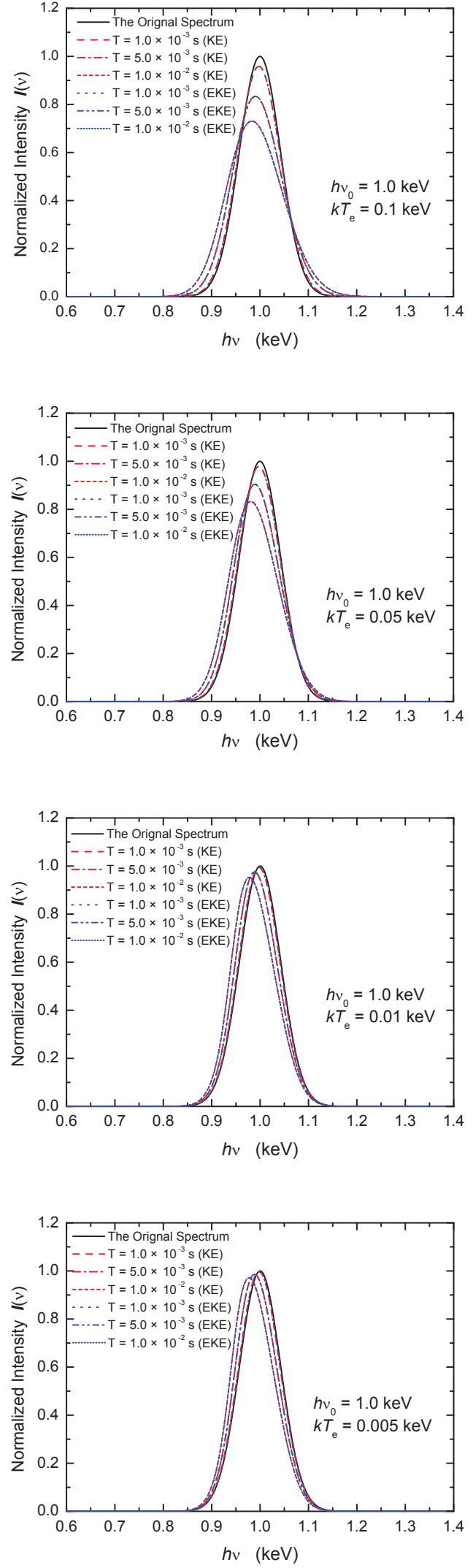

Fig. 2. Comparison of evolution behavior for the down-Comptonization of the Gaussian line with $h v_{0}=1 \mathrm{keV}$ between the classical Kompaneets equation and our extended equation. The evolution times $T, h v_{0}$, and $k T_{\mathrm{e}}$ are labeled in the figures. The blue and red lines represent the calculated results of the extended equation and the Kompaneets equation, respectively.
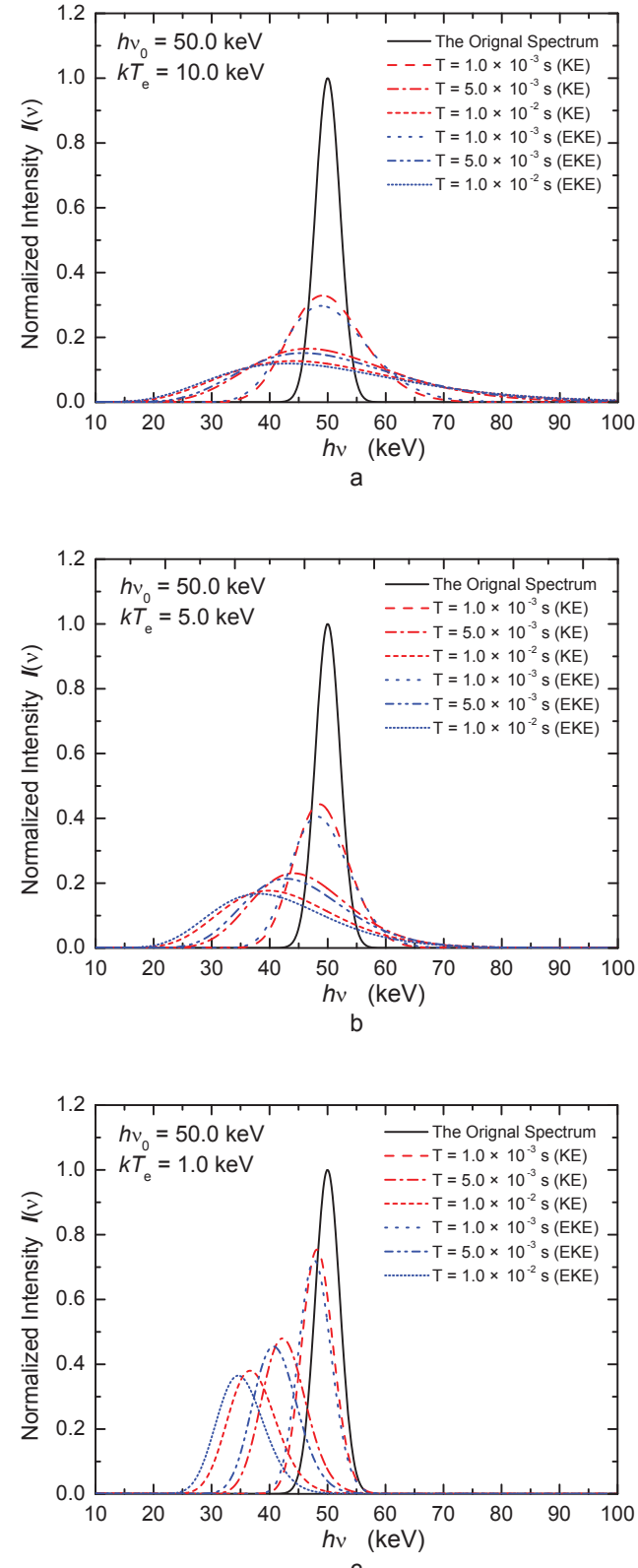

Fig. 3. Comparison of evolution behavior for the down-Comptonization of the Gaussian line with $h v_{0}=50 \mathrm{keV}$ between the classical Kompaneets equation and our extended equation. The evolution times $T, h v_{0}$, and $k T_{\mathrm{e}}$ are labeled in the figures. The blue and red lines represent the calculated results of the extended equation and the Kompaneets equation, respectively.

\section{The Sunyaev-Zel'dovich effect correctness}

The Sunyaev-Zel'dovich effect (SZE; Birkinshaw 1999; Carlstrom 2002; Rephaeli 1995; Challinor \& Lasenby 1998) causes a change in the apparent brightness of the cosmic microwave background (CMB) when seen through the hot gas in a cluster of galaxies or any other reservoir of hot plasma. The SZE has a unique spectral signature that shows a decrease in the cosmic microwave background intensity at frequencies lower than around $218 \mathrm{GHz}$, and an increase in the intensity at higher frequencies. Clusters discovered by the SZE will determine their redshifts, mass content, and other properties. Just recently, an approach was proposed by Titarchuk \& Lipunova (2019) to 
determine the optical depth and the electron temperature of a cluster by fitting the observation results of $\mathrm{CMB}$ through the plasma cloud.

The SZE usually employs the classical Kompaneets equation, with nonrelativistic treatments (Birkinshaw 1999). The unsimplified change in intensity is

$\Delta I(v)=I_{0} \tau_{\mathrm{c}} \frac{k T_{\mathrm{e}}}{m_{\mathrm{e}} c^{2}}\left(1-\frac{T_{\mathrm{rad}}}{T_{\mathrm{e}}}\right) \frac{X^{4} e^{X}}{\left(e^{X}-1\right)^{2}}\left[X \frac{e^{X}+1}{e^{X}-1}-4\right]$,

where the capital $X, X \equiv h v / k T_{\text {rad }}$, is different from the previous definition of the small letter $x, x \equiv h v / k T_{\mathrm{e}} ; k T_{\mathrm{rad}}$ is the equivalent temperature of photon gas (or radiation field) and $I_{0}=\frac{2 h}{c^{2}}\left(\frac{k T_{\mathrm{rad}}}{h}\right)^{3} ;$ and $\tau_{\mathrm{c}}=\int N_{\mathrm{e}}(r) \sigma_{\mathrm{T}} \mathrm{d} r$ represents the optical depth (see $I_{0}$ and $\tau_{\mathrm{c}}$ in Birkinshaw 1999; Molnar \& Birkinshaw 1999), which is treated as a constant value here.

Recently, re-deriving the SZE from a more precise equation was tried by some authors (Challinor \& Lasenby 1998; Itoh et al. 1998; Stebbins 1997). If using our extended Kompaneets equation Eq. (35), the modified SZE should be

$$
\begin{aligned}
\frac{\Delta I(v)}{I_{0} \tau_{\mathrm{c}}}= & \frac{k T_{\mathrm{e}}}{m_{\mathrm{e}} c^{2}}\left(1-\frac{T_{\mathrm{rad}}}{T_{\mathrm{e}}}\right) \frac{X^{4} e^{X}}{\left(e^{X}-1\right)^{2}} X \\
& \times\left[\left(1+\frac{14}{5} \frac{k T_{\mathrm{rad}}}{m_{\mathrm{e}} c^{2}} X\right) \frac{e^{X}+1}{e^{X}-1}-4-14 \frac{k T_{\mathrm{rad}}}{m_{\mathrm{e}} c^{2}}\right] .
\end{aligned}
$$

This modified SZE works for both cases, $k T_{\text {rad }}<k T_{\mathrm{e}} \ll$ $m_{\mathrm{e}} c^{2}$ and $k T_{\mathrm{e}}<k T_{\text {rad }} \ll m_{\mathrm{e}} c^{2}$, so the comparison between $k T_{\text {rad }}$ and $k T_{\mathrm{e}}$ is no longer required, which is an obvious advantage for this SZE, differing from the other modified SZEs. Comparing with Eq. (39), the two new terms $\left(1+\frac{14}{5} \frac{k T_{\mathrm{rad}}}{m_{\mathrm{e}} c^{2}} X\right)$ and $\left(-14 \frac{k T_{\mathrm{rad}}}{m_{\mathrm{e}} c^{2}}\right)$ are included in Eq. (40) and both depend on $T_{\text {rad }}$. However, for the $\mathrm{CMB}$ when passing through hot clusters where $k T_{\mathrm{e}}$ is greater than $\sim 1 \mathrm{keV}$, due to $T_{\mathrm{rad}} \ll k T_{\mathrm{e}} \ll m_{\mathrm{e}} c^{2}$, the SZE Eqs. (39) and (40) are approximately identical:

$$
\frac{\Delta I(v)}{I_{0} \tau_{\mathrm{c}}}=\frac{k T_{\mathrm{e}}}{m_{\mathrm{e}} c^{2}} \frac{X^{4} e^{X}}{\left(e^{X}-1\right)^{2}}\left[X \frac{e^{X}+1}{e^{X}-1}-4\right] .
$$

In general, the temperatures of hot electron gas in clusters are much higher: $k T_{\mathrm{e}} \simeq 10 \sim 20 \mathrm{keV}$. For example, for the A2163 Cluster, $k T_{\mathrm{e}} \simeq 12.4 \pm 0.5 \mathrm{keV}$ (Sarazin 1986; Rosati et al. 2002). Therefore, we compute the change in intensity of the blackbody spectrum with $k T_{\text {rad }}=2.37 \times 10^{-7} \mathrm{keV}(\mathrm{CMB})$ shown in Fig. 4 for thermal electron gas at $k T_{\mathrm{e}}=5 \mathrm{keV}, 10 \mathrm{keV}$, and $25 \mathrm{keV}$. Figure 4 shows that the discrepancy between the classical Kompaneets equation and our newly obtained Eq. (35) can be neglected for the Comptonization of CMB when passing through hot electron gas in the clusters, just because the radiation temperature of CMB is too low, $k T_{\text {rad }} \ll m_{\mathrm{e}} c^{2}$, for which Eq. (40) is almost exactly same as Eq. (39), i.e., the extended equation Eq. (35) returns to the Kompaneets equation Eq. (2).

Although our extended Kompaneets equation obviously returns to the classical Kompaneets equation in the CMB condition, the differences of the evolution behavior between the classical Kompaneets equation and our new equation are outstanding for much higher temperature blackbody spectrum $k T_{\text {rad }}>10 \mathrm{keV}$ when passing through the cold plasma surrounding the X-ray sources, shown in the following figures. Many authors have mentioned the radiation transfer process (i.e., the Comptonization) for X-ray and $\gamma$-ray photons including continuum spectra and emission lines in luminous sources, such as the compact X-ray binary sources, the supernova remnants, and

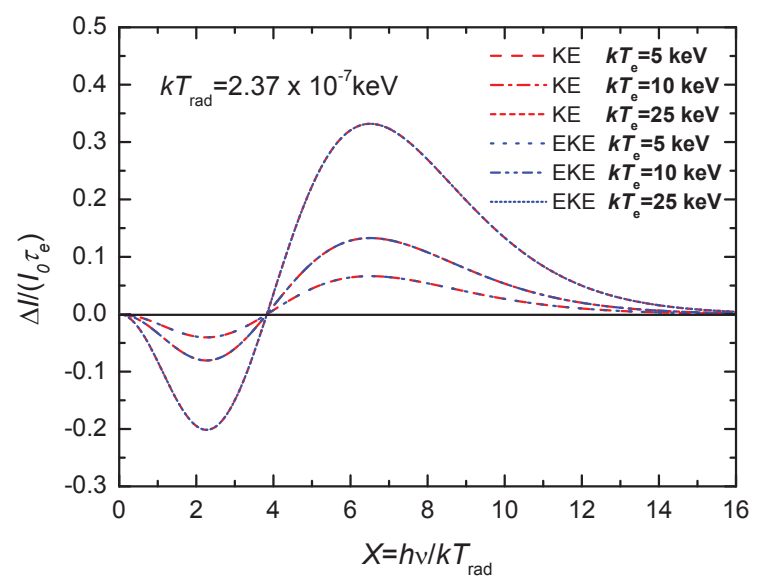

Fig. 4. Change in intensity of the blackbody spectrum with $k T_{\text {rad }}=$ $2.35 \times 10^{-7} \mathrm{keV}(\mathrm{CMB})$ for thermal electron gas at $k T_{\mathrm{e}}=5 \mathrm{keV}, 10 \mathrm{keV}$, and $25 \mathrm{keV}$ as a function of dimensionless frequency $X=h v /\left(k T_{\mathrm{rad}}\right)$, with scaling $I_{0} y_{\mathrm{c}}=\frac{2 h}{c^{2}}\left(\frac{k T_{\text {rad }}}{h}\right)^{3} y_{\mathrm{c}}$. The results obtained from the extended Kompaneets equation are shown as blue lines, while the red lines denote the results from the classical Kompaneets equation.

the active galactic nuclei (Ross et al. 1978; Misra \& Kembhavi 1998; Zhang et al. 2000; Wang et al. 2014; Adegoke et al. 2019; McCray \& Hatchett 1975; Seward et al. 2012). In addition, the CMB is a pretty blackbody spectrum in our universe; the blackbody spectrum of X-rays is given by the Planckian formula in the following form, which is used in the obtainment of Eq. (39),

$I(v)=B_{v}\left(T_{\mathrm{rad}}\right)=\frac{2 h v^{3}}{c^{2}} \cdot \frac{1}{\exp \left[h v / k T_{\mathrm{rad}}\right]-1}$.

To be specific, in the following model calculations, we take the typical temperatures of the X-ray blackbody spectrum, $k T_{\text {rad }}=1 \mathrm{keV}, k T_{\text {rad }}=5 \mathrm{keV}$, and $k T_{\text {rad }}=10 \mathrm{keV}$. In addition to calculating the up-Comptonization of X-ray blackbody spectra, we show the evolution behavior of blackbody spectrum under the extended Kompaneets equation and the classical Kompaneets equation, particularly in order to point out the distinct difference between the extended Kompaneets equation and the Kompaneets equation for dealing with the down-Comptonization of highenergy blackbody photons. The down-Comptonization of the $\mathrm{X}$-ray blackbody spectrum will be the main focus; therefore, in the calculations we take $k T_{\mathrm{e}} \leq k T_{\text {rad }}$ to ensure the possibility of down-Comptonization, particularly at the high-energy side of the blackbody spectrum. The initial condition now becomes

$n(x, 0)=f(x) \sim \frac{1}{\exp \left[x \cdot\left(T_{\mathrm{e}} / T_{\mathrm{rad}}\right)\right]-1}$.

When $k T_{\mathrm{e}}<k T_{\text {rad }}$, the calculated evolution behavior of blackbody spectrum for $k T_{\text {rad }}=1 \mathrm{keV}, k T_{\text {rad }}=5 \mathrm{keV}$, and $k T_{\text {rad }}=10 \mathrm{keV}$ are shown in Figs. 5, 6, and A.8, respectively. If $k T_{\mathrm{e}}>k T_{\mathrm{rad}}$, the calculated evolution results for blackbody spectrum $k T_{\text {rad }}=1 \mathrm{keV}, k T_{\text {rad }}=5 \mathrm{keV}$, and $k T_{\text {rad }}=10 \mathrm{keV}$ are respectively shown in Figs. A.6, A.9, and A.11. The blue lines represent the calculated results by our extended Kompaneets equation, while the red lines represent the calculated results by the classical Kompaneets equation, the temperatures of electron gas are labeled with different lines in each figure. At the same time, for comparison between the newly obtained equation and the Kompaneets equation, we also compute the change in intensity of the blackbody spectrum with $k T_{\text {rad }}=1 \mathrm{keV}$, 
$k T_{\text {rad }}=5 \mathrm{keV}$, and $k T_{\text {rad }}=10 \mathrm{keV}$ using Eqs. (39) and (40) shown respectively in Figs. A.7, A.10, and 7 under the same temperatures of thermal electron gas as above. The spectral evolution behavior of blackbody spectrum are revealed as in the description of the Gaussian lines in Sect. 3. It should be noted that the big differences between the evolution behavior of high-energy blackbody photons (e.g., $k T_{\text {rad }}>10 \mathrm{keV}$ ) obtained by the extended Kompaneets equation and the original Kompaneets equation are clearly exhibited in Figs. 6 and A.11. Thus, we point out that we should use the extended Eq. (35) to deal with the Comptonization of high-energy photons including up-Comptonization and down-Comptonization instead of Eq. (2) because our equation is obtained under much looser conditions $h v \ll m_{\mathrm{e}} c^{2}$ and $k T_{\mathrm{e}} \ll m_{\mathrm{e}} c^{2}$ than $h v \ll k T_{\mathrm{e}} \ll m_{\mathrm{e}} c^{2}$ for Eq. (2).

\section{Conclusions and discussion}

In this study, differing from the method used to obtain Eq. (4) by Liu et al. (2004), we present a new extended Kompaneets equation (35) by expanding the distribution function in $\Delta p$ instead of $\Delta v$, which can be used to describe a more general Comptonization process, including down- and up-Comptonization, suitable for any case, $h v \ll k T_{\mathrm{e}}, h v \gg k T_{\mathrm{e}}$, and $h v \sim k T_{\mathrm{e}}$. The condition $h v \ll k T_{\mathrm{e}}$, under which the original Kompaneets equation (2) was derived, is no longer necessary for Eq. (35). The Kompaneets equation cannot give an accurate description of the down-Comptonization $\left(h v \gg k T_{\mathrm{e}}\right)$ for Xrays and $\gamma$ rays passing through the cold plasma, which is the most important radiative transfer process in high-energy astronomy (Ross et al. 1978; O’Dell 1986; Misra \& Kembhavi 1998; Reynolds 2008; Kallman et al. 1979). However, our new extended equation will have potential applications in dealing with the Comptonization process in astrophysics including down- and up-Comptonization, especially for the downComptonization of hard X-rays and $\gamma$ rays (see the calculated results in Sects. 3 and 4).

By Eq. (35) we give some typical numerical solutions including the evolution behavior of photons with Gaussian-line and blackbody spectra in X-ray and $\gamma$-ray astronomy, and compare them with the results of Kompaneets equation in Sects. 3 and 4. From these results, the significant changes of the emergent radiation spectrum from plasmas due to the affection of Comptonization have been shown in the calculated spectral curves (see figures in Sects. 3, 4, and Appendix A). Therefore, Comptonization as one of the most important radiative transfer processes in high-energy astrophysics should be noted (see Adegoke et al. 2019, and references therein). Above all things, our calculations show that for the photons with much lower energy than $m_{\mathrm{e}} c^{2}$ (i.e., $h v \ll m_{\mathrm{e}} c^{2}$ ) there is excellent consistency between the Comptonevolution spectra obtained by the use of the extended Kompaneets equation and the original Kompaneets equation, which confirms the correctness of our extended Kompaneets equation.

In addition, our calculations show that the change in the emergent spectrum in Comptonization, particularly in downComptonization, depends on the difference between $h \bar{v}$ and $k T_{\mathrm{e}}$ and on the scattering depth $\tau_{\mathrm{s}}$ (or, equivalently, the diffusion timescale $T$ ): the larger the difference $h \bar{v}-k T_{\mathrm{e}}$, and/or the larger the depth $\tau_{\mathrm{s}}$ (equivalently, the timescale $T$ ), the larger the change in the emergent spectrum. In addition, the Compton-evolution spectra for the blackbody spectrum and the Gaussian line have similar spectral evolution behavior. In particular, for both the blackbody spectrum and the Gaussian line, if only the energy of photons is high enough (e.g., $h v>10 \mathrm{keV}$ ), there are big differences between the evolution behavior obtained via the extended
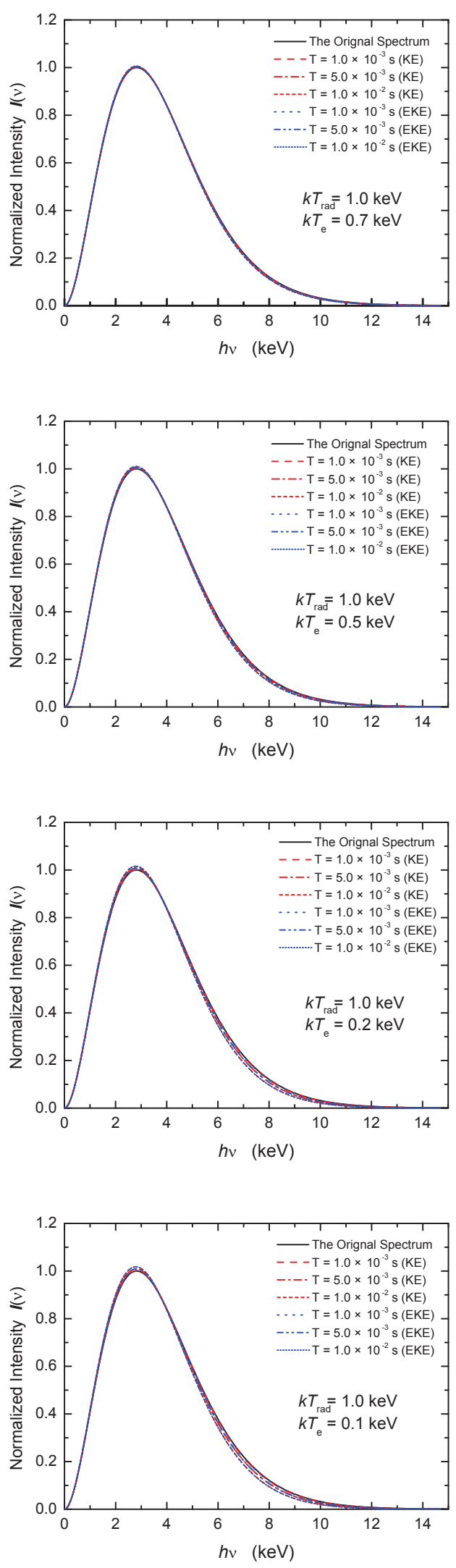

Fig. 5. Comparison of evolution behavior for the down-Comptonization of X-ray blackbody spectrum with $k T_{\text {rad }}=1 \mathrm{keV}$ between the classical Kompaneets equation and our extended equation. The evolution times $T, k T_{\text {rad }}$, and $k T_{\mathrm{e}}$ are labeled in the figures. The blue and red lines represent the calculated results of the extended equation and the Kompaneets equation, respectively. 

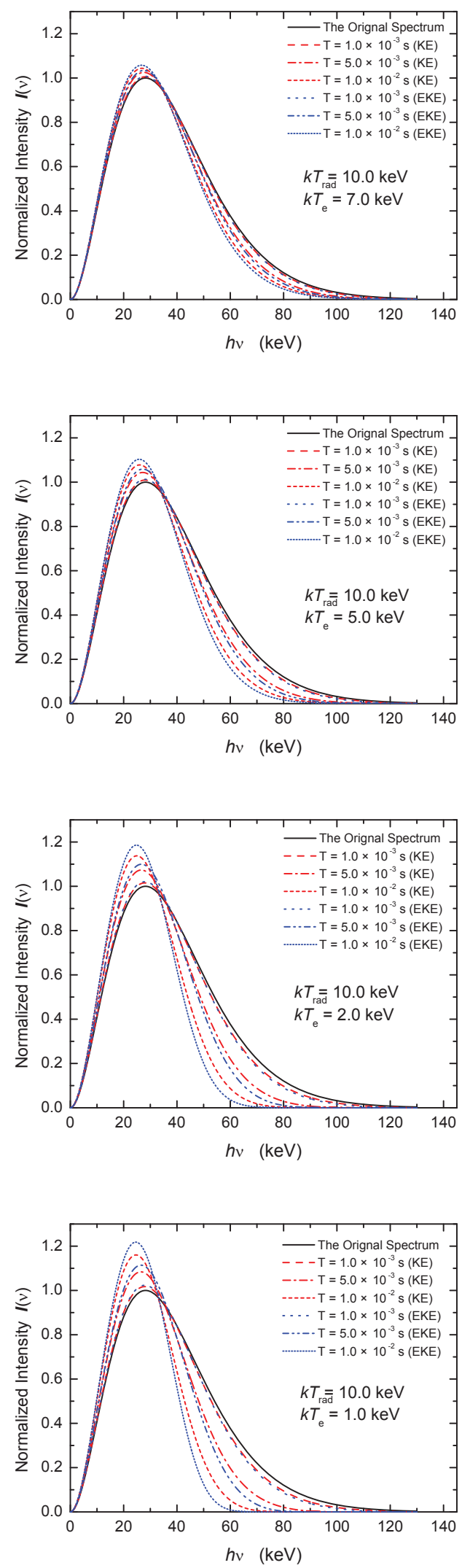

Fig. 6. Comparison of evolution behavior for the down-Comptonization of X-ray blackbody spectrum with $k T_{\text {rad }}=10 \mathrm{keV}$ between the classical Kompaneets equation and our extended equation. The evolution times $T, k T_{\text {rad }}$, and $k T_{\mathrm{e}}$ are labeled in the figures. The blue and red lines represent the calculated results of the extended equation and the Kompaneets equation, respectively.
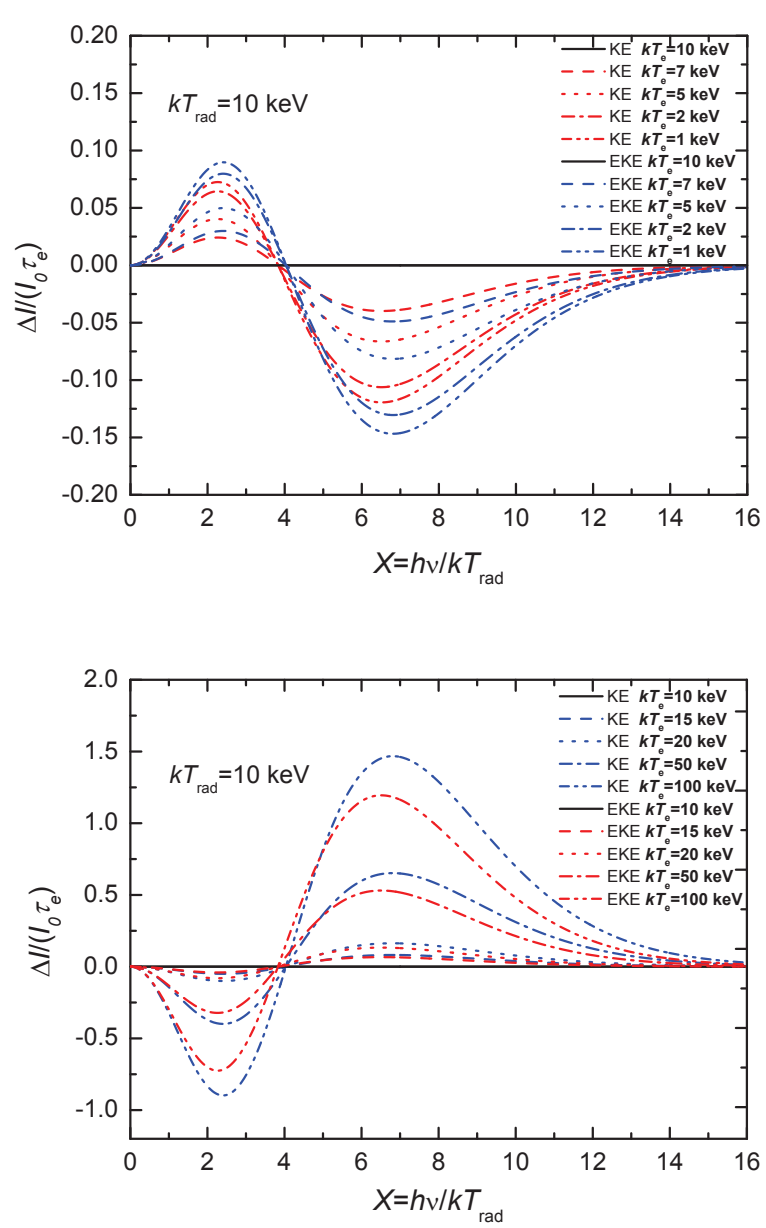

Fig. 7. Change in intensity of the blackbody spectrum with $k T_{\mathrm{rad}}=$ $10 \mathrm{keV}$ under different temperatures of thermal electron gas calculated by use of Eqs. (39) and (40) as a function of dimensionless frequency $X=h v /\left(k T_{\text {rad }}\right)$, with scaling $I_{0} y_{\mathrm{c}}=\frac{2 h}{c^{2}}\left(\frac{k T_{\mathrm{rad}}}{h}\right)^{3} y_{\mathrm{c}} \cdot k T_{\text {rad }}$ and $k T_{\mathrm{e}}$ are labeled in the figures. The results obtained under the extended Kompaneets equation and the classical Kompaneets equation are shown as blue and red lines, respectively.

Kompaneets equation and via the original Kompaneets equation (see Figs. 3, 6, 7, A.4, and A.11), which indicates that we should use the extended Eq. (35) to deal with the Comptonization of high-energy photons including up-Comptonization and downComptonization instead of Eq. (2) because the term $\frac{14}{5} \frac{k T_{\mathrm{e}}}{m_{\mathrm{e}} c^{2}} x$ in Eq. (35) can bring a significant contribution to the evolution behavior for the high-energy photons. Therefore, Eq. (22) can be regarded as an important improvement of the original Kompaneets equation (2), particularly for hard X-ray and $\gamma$-ray astronomy.

The structure of Eq. (35) has two marked characteristics that can be regarded as a criterion of the correctness of our Eq. (35). First, it has the form $\frac{1}{x^{2}} \frac{\partial}{\partial x}\left\{x^{4}\left(1+\frac{14}{5} \frac{k T_{\mathrm{e}}}{m_{\mathrm{e}} c^{2}} x\right)\left[\frac{\partial n}{\partial x}+n(n+1)\right]\right\}$, which ensures the conservation of number of photons (see the conservation Eq. (28)). The invariance of the total number of photons is a basic requirement for the electron-photon scattering process. Second, Eq. (35) contains a factor $\left[\frac{\partial n}{\partial x}+n(n+1)\right]$, which ensures $\partial n / \partial t=0$ when the photon gas reaches the thermal equilibrium distribution $n=\left(e^{x}-1\right)^{-1}$. This is also a necessary requirement for the correctness of this equation because the 
thermal equilibrium will inevitably be reached through the scattering and $\partial n / \partial t \rightarrow 0$, which implies that the diffusion finally stops. Ross et al. (1978) also noticed the restriction $h v \ll k T_{\mathrm{e}} \ll$ $m_{\mathrm{e}} c^{2}$ of the original Kompaneets equation (2) and suggested an alternative equation to replace Eq. (2) to suit the case $h \bar{v} \gg k T_{\mathrm{e}}$. Their equation has the form $\frac{1}{x^{2}} \frac{\partial}{\partial x}\left\{x^{4}\left[n+\left(1+\frac{7}{10} \frac{k T_{\mathrm{e}}}{m_{\mathrm{e}} c^{2}} x^{2}\right) \frac{\partial n}{\partial x}\right]\right\}$, which is quite similar to Eq. (35), but deviates from the necessary form $\left[\frac{\partial n}{\partial x}+n(n+1)\right]$. According to their equation, the diffusion will never stop, i.e., $\partial n / \partial t \neq 0$ even when the thermal equilibrium, $n=\left(e^{x}-1\right)^{-1}$, has been reached.

As a result, we note again that our extended Kompaneets equation obviously returns to the classical Kompaneets equation in the CMB condition (i.e., the Sunyaev-Zel'dovich effect (SZE)), and Eqs. (39) and (40) are approximately identical for CMB. However, just as indicated by many authors, Compton up-scattering of optical-UV seed photons into X-rays in the hot corona can provide a compelling explanation for the optical-UV-X-ray correlated variability seen in AGN and compact X-ray binaries (Ross et al. 1978; Zhang et al. 2000; Gaskell 2007; Breedt et al. 2010; Fabian et al. 2015; Buisson et al. 2018; Bonson et al. 2018; Adegoke et al. 2019). Recently, Adegoke et al. (2019) have argued that there is a UV to X-ray Comptonization delay in the narrow-line Seyfert 1 galaxy Mrk 493. Through the light variations in the UV emission preceding the variations in the X-ray emission based on $\sim 100 \mathrm{ks} X M M-N e w t o n$ observations of Mrk 493, they found that the UV emission leads by $\sim 5 \mathrm{ks}$ relative to the X-ray emission. Then, they reported that the UV lead is consistent with the time taken by the UV photons to travel from the location of their origin in the accretion disk to the hot corona, and the time required for repeated inverse Compton scattering converting the UV photons into X-ray photons. In addition, for X-ray binaries, Zhang et al. (2000) have proposed the threelayered atmospheric structure in accretion disks around stellarmass black holes. Also, the Comptonization process may be operating in these systems. Therefore, our extended equation will have potential applications for dealing with the Comptonization process in astrophysics including down- and up-Comptonization, especially for the down-Comptonization of hard X-rays and $\gamma$ rays (see the calculated results in Sects. 3 and 4). In the future, we plan to investigate the Comptonization process in high-energy astrophysics, especially for X-ray and $\gamma$-ray astronomy.

Acknowledgements. We thank J. Ling and J. Evslin for fruitful discussions and suggestions. This work is partly supported by the Strategic Priority Research
Program of Chinese Academy of Sciences, Grant No. XDB34030301. DL acknowledges support by the National Science Foundation of China (Grant Nos. U1631101, 11665022, 11233006), and the Shanghai Science and Technology Commission (Grant No. 16ZR1417200).

\section{References}

Adegoke, O., Dewangan, G. C., Pawar, P., et al. 2019, ApJ, 870, L13 Birkinshaw, M. 1999, Phys. Rep., 310, 97

Breedt, E., McHardy, I. M., Arevalo, P., et al. 2010, MNRAS, 403, 605

Bonson, K., Gallo, L. C., Wilkins, D. R., et al. 2018, MNRAS, 477, 3247

Buisson, D. J. K., Fabian, A. C., \& Lohfink, A. M. 2018, MNRAS, 481, 4419

Carlstrom, J. E. 2002, ARA\&A, 40, 643

Challinor, A., \& Lasenby, A. 1998, ApJ, 499, 1

Chen, J. F., You, J. H., \& Cheng, F. H. 1994, J. Phys. A: Math. Gen., 27, 2905

Deng, J. S., Chen, J. F., \& You, J. H. 1998, Sci. China (Ser. A), 41, 10

Fabian, A. C., Nandra, K., Reynolds, C. S., et al. 1995, MNRAS, 277, L11

Fabian, A. C., Lohfink, A., Kara, E., et al. 2015, MNRAS, 451, 4375

Felten, J. E., \& Rees, M. J. 1972, A\&A, 17, 226

Gaskell, C. M. 2007, Proc. ASP Conf. Ser., eds. L. C. Ho, \& J.-M. Wang, 373, 596

Hua, X. M., Kazanas, D., \& Titarchuk, L. G. 1997, ApJ, 482, L57

Illarionov, A. F., Kallman, T., McCray, R., et al. 1979, ApJ, 228, 279

Itoh, N., Kohyama, Y., \& Nozawa, S. 1998, ApJ, 502, 7

Kallman, T., McCray, R., \& Ross, R. R. 1979, ApJ, 228, 279

Kazanas, D., Hua, X. M., \& Titarchuk, L. G. 1997, ApJ, 480, 735

Kompaneets, A. S. 1957, Sov. Phys. JETP, 4, 730

Kompaneets, A. S., \& Eksper, Z. 1956, Teoret. Fiz., 31, 876

Liu, D. B., Chen, L., Ling, J. J., et al. 2004, A\&A, 417, 381

McCray, R., \& Hatchett, S. 1975, ApJ, 199, 196

Misra, R., \& Kembhavi, A. K. 1998, ApJ, 499, 205

Miyamoto, S. 1978, A\&A, 63, 69

Molnar, S. M., \& Birkinshaw, M. 1999, ApJ, 523, 78

O’Dell, S. L. 1986, PASP, 98, 140

Rephaeli, Y. 1995, ApJ, 445, 33

Reynolds, S. P. 2008, ARA\&A, 46, 89

Rybicki, G. B., \& Lightman, A. P. 1979, Radiative Processes in Astrophysics (New York: Wiley)

Rosati, P., Borgani, S., \& Norman, C. 2002, ARA\&A, 40, 539

Ross, R. R., Weaver, R., \& McCray, R. 1978, ApJ, 219, 292

Sarazin, C. L. 1986, Rev. Mod. Phys., 58, 1

Seward, F. D., Charles, P. A., Foster, D. L., et al. 2012, ApJ, 759, 123

Shakura, N. I., \& Sunyaev, R. A. 1973, A\&A, 24, 337

Stebbins, A. 1997, ArXiv e-prints [arXiv:astro-ph/9705178]

Sunyaev, R. A., \& Titarchuk, L. G. 1980, A\&A, 86, 121

Sunyaev, R. A., \& Titarchuk, L. G. 1985, A\&A, 143, 374

Titarchuk, L. G. 1994, ApJ, 434, 570

Titarchuk, L. G., \& Lipunova, G. V. 2019, ArXiv e-prints [arXiv:1906.07060]

Treves, A., Morini, M., Chiappetti, L., et al. 1989, ApJ, 341, 733

Wang, J. M., Du, P., Hu, C., et al. 2014, ApJ, 793, 108

Zhang, S. N., Cui, W., Chen, W., et al. 2000, Science, 287, 1239 


\section{Appendix A: Additional figures}

In this appendix we present the figures regarding the behavior of Compton evolution behavior of different spectra as a supplement to the main text, where the conclusions and discussions of Comptonization under various conditions are given. In Figs. A.1-A.5 we compare the evolution behavior of Gaussian line spectra with $h v_{0}=1.0 \mathrm{keV}, 10 \mathrm{keV}$ for the up-Compotnization and $h v_{0}=10 \mathrm{keV}, 100 \mathrm{keV}, 200 \mathrm{keV}$ for the down-Comptonization. Moreover, Figs. A.6-A.11 show the Comptonization of X-ray blackbody spectra with $k T_{\text {rad }}=$ $1.0 \mathrm{keV}, 5.0 \mathrm{keV}, 10 \mathrm{keV}$ for both up- and down-Comptonization. The intensity changes obtained under classical and extended Kompaneets equations are also displayed here.
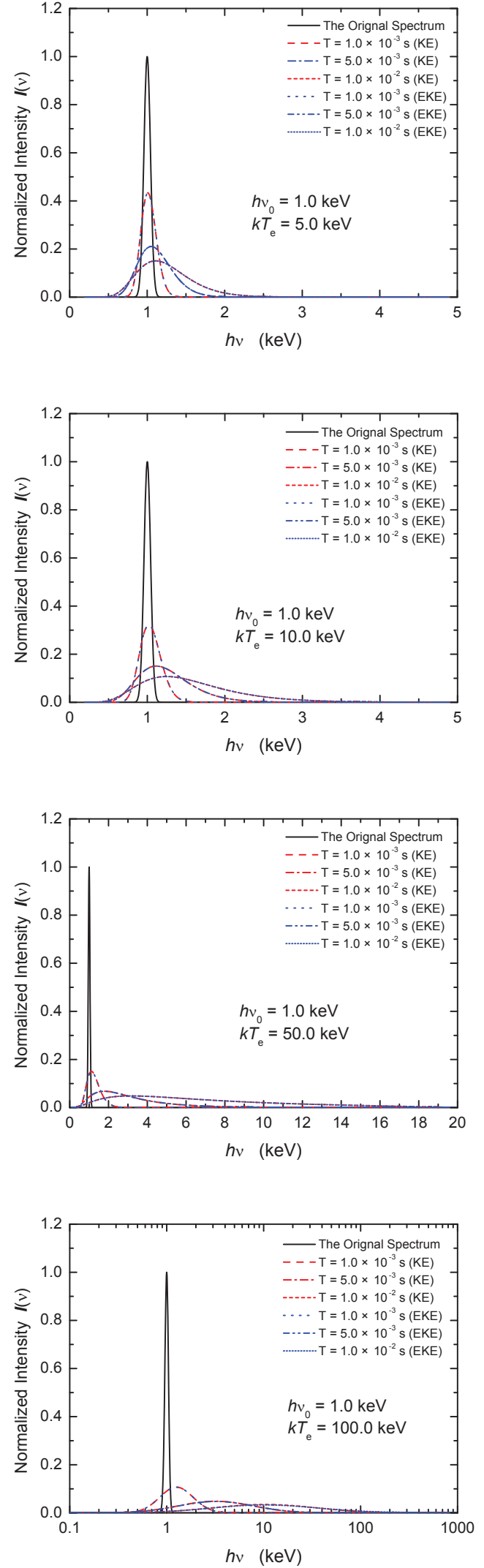

Fig. A.1. Comparison of evolution behavior for the up-Comptonization of the Gaussian line with $h v_{0}=1 \mathrm{keV}$ between the classical Kompaneets equation and our extended equation. The evolution times $T, h v_{0}$, and $k T_{\mathrm{e}}$ are labeled in the figures. The blue and red lines represent the calculated results of the extended equation and the Kompaneets equation, respectively. 
X. Chen et al.: Modified form of the Kompaneets equation
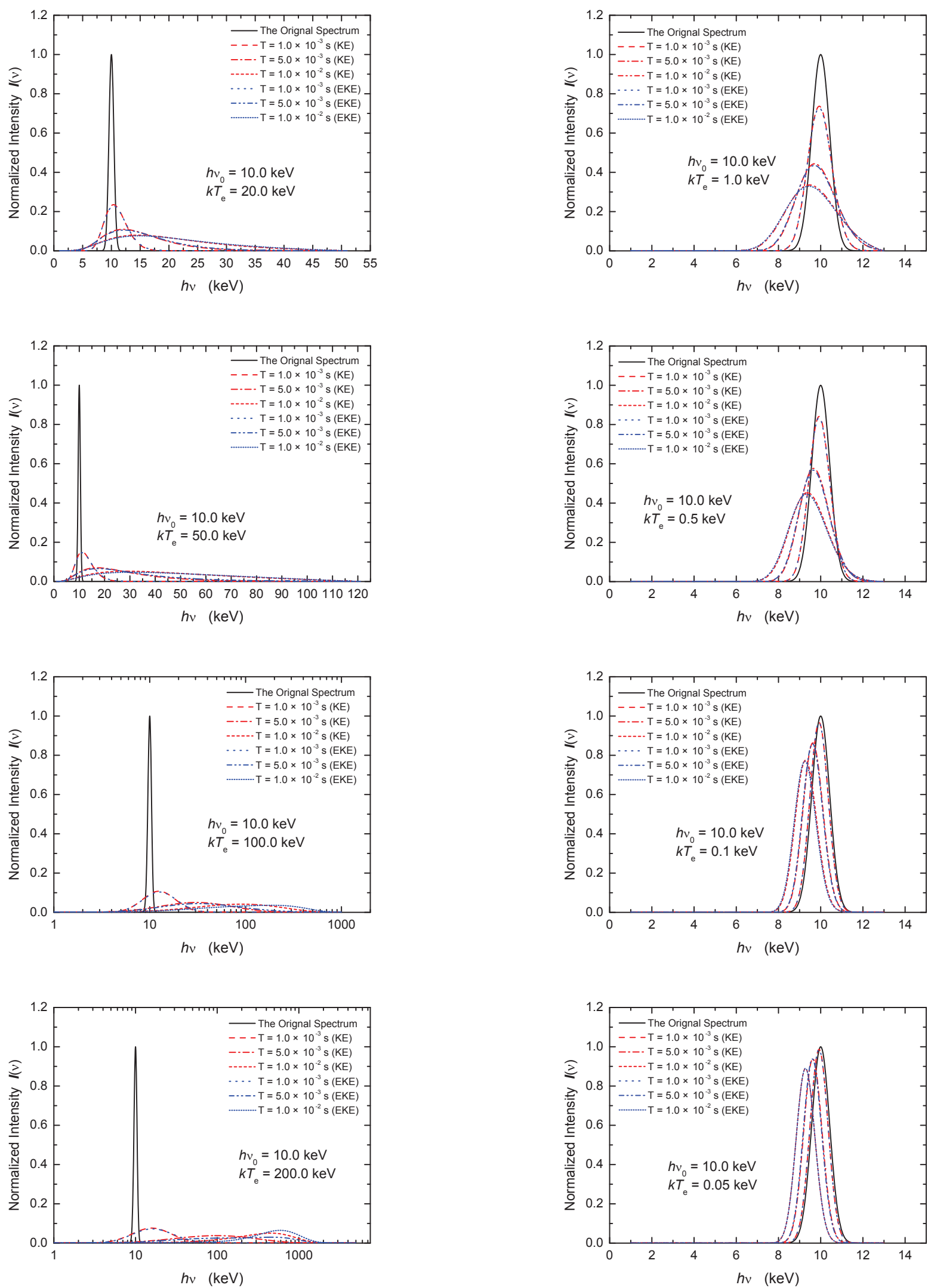

Fig. A.2. Comparison of evolution behavior for the up-Comptonization of the Gaussian line with $h v_{0}=10 \mathrm{keV}$ between the classical Kompaneets equation and our extended equation. The evolution times $T, h v_{0}$, and $k T_{\mathrm{e}}$ are labeled in the figures. The blue and red lines represent the calculated results of the extended equation and the Kompaneets equation, respectively.

Fig. A.3. Comparison of evolution behavior for the downComptonization of the Gaussian line with $h v_{0}=10 \mathrm{keV}$ between the classical Kompaneets equation and our extended equation. The evolution times $T, h v_{0}$, and $k T_{\mathrm{e}}$ are labeled in the figures. The blue and red lines represent the calculated results of the extended equation and the Kompaneets equation, respectively. 

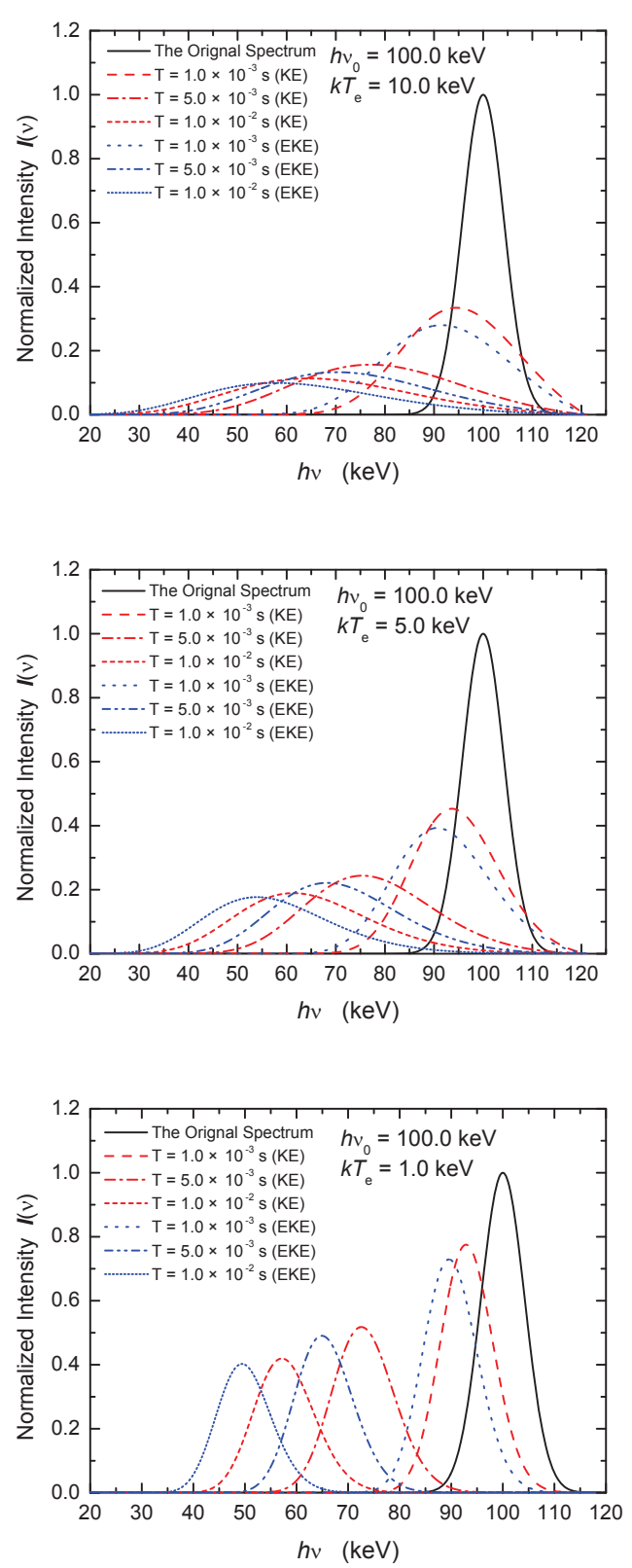

Fig. A.4. Comparison of evolution behavior for the downComptonization of the Gaussian line with $h v_{0}=100 \mathrm{keV}$ between the classical Kompaneets equation and our extended equation. The evolution times $T, h v_{0}$, and $k T_{\mathrm{e}}$ are labeled in the figures. The blue and red lines represent the calculated results of the extended equation and the Kompaneets equation, respectively.
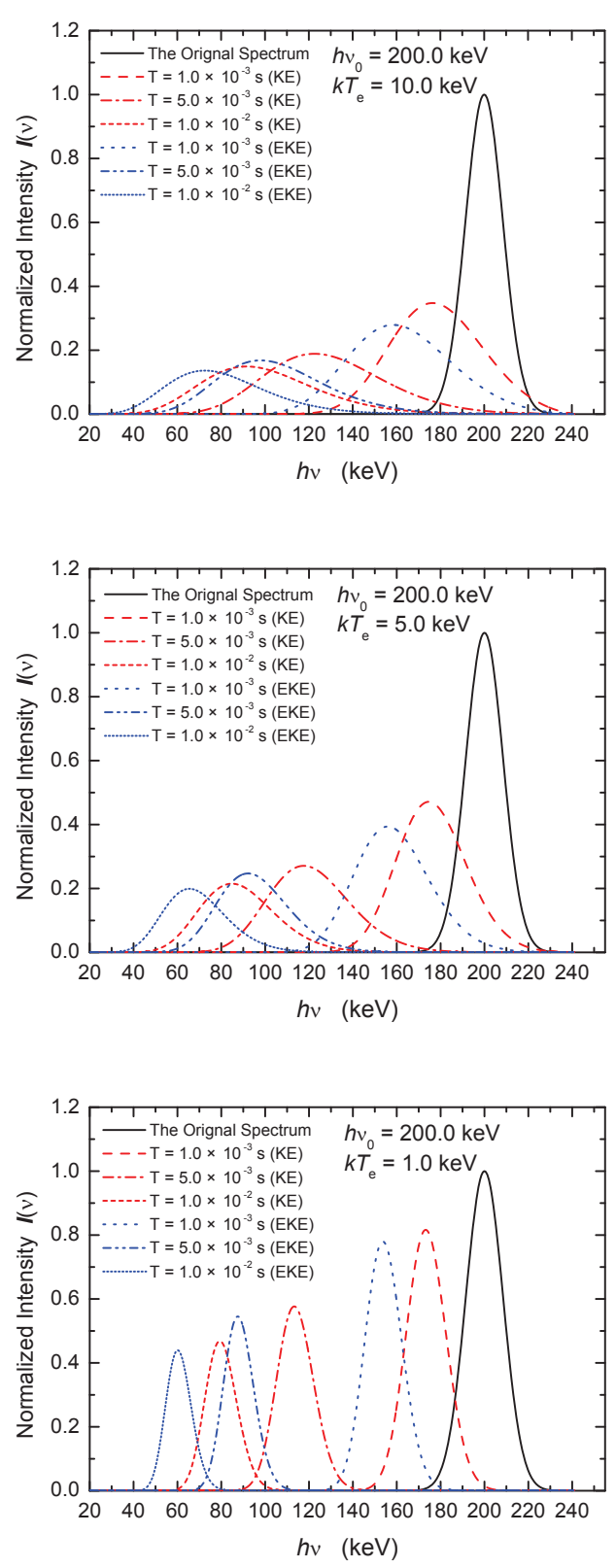

Fig. A.5. Comparison of evolution behavior for the downComptonization of the Gaussian line with $h v_{0}=200 \mathrm{keV}$ between the classical Kompaneets equation and our extended equation. The evolution times $T, h v_{0}$, and $k T_{\mathrm{e}}$ are labeled in the figures. The blue and red lines represent the calculated results of the extended equation and the Kompaneets equation, respectively. 
X. Chen et al.: Modified form of the Kompaneets equation
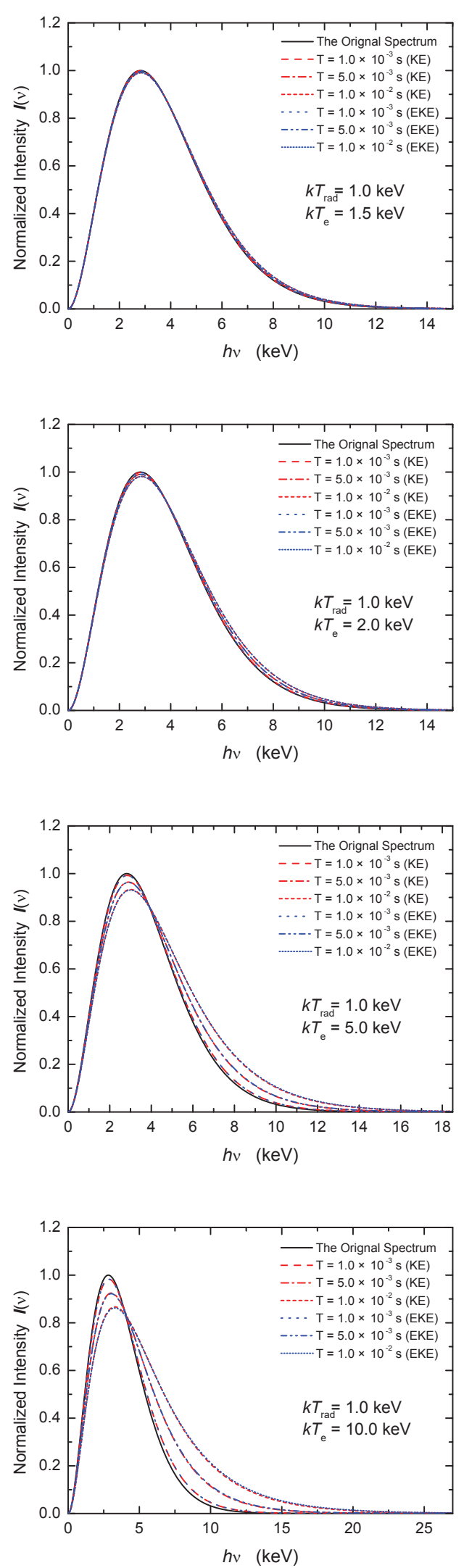

Fig. A.6. Comparison of evolution behavior for the up-Comptonization of X-ray blackbody spectrum with $k T_{\text {rad }}=1 \mathrm{keV}$ between the classical Kompaneets equation and our extended equation. The evolution times $T, k T_{\text {rad }}$, and $k T_{\mathrm{e}}$ are labeled in the figures. The blue and red lines represent the calculated results of the extended equation and the Kompaneets equation, respectively.
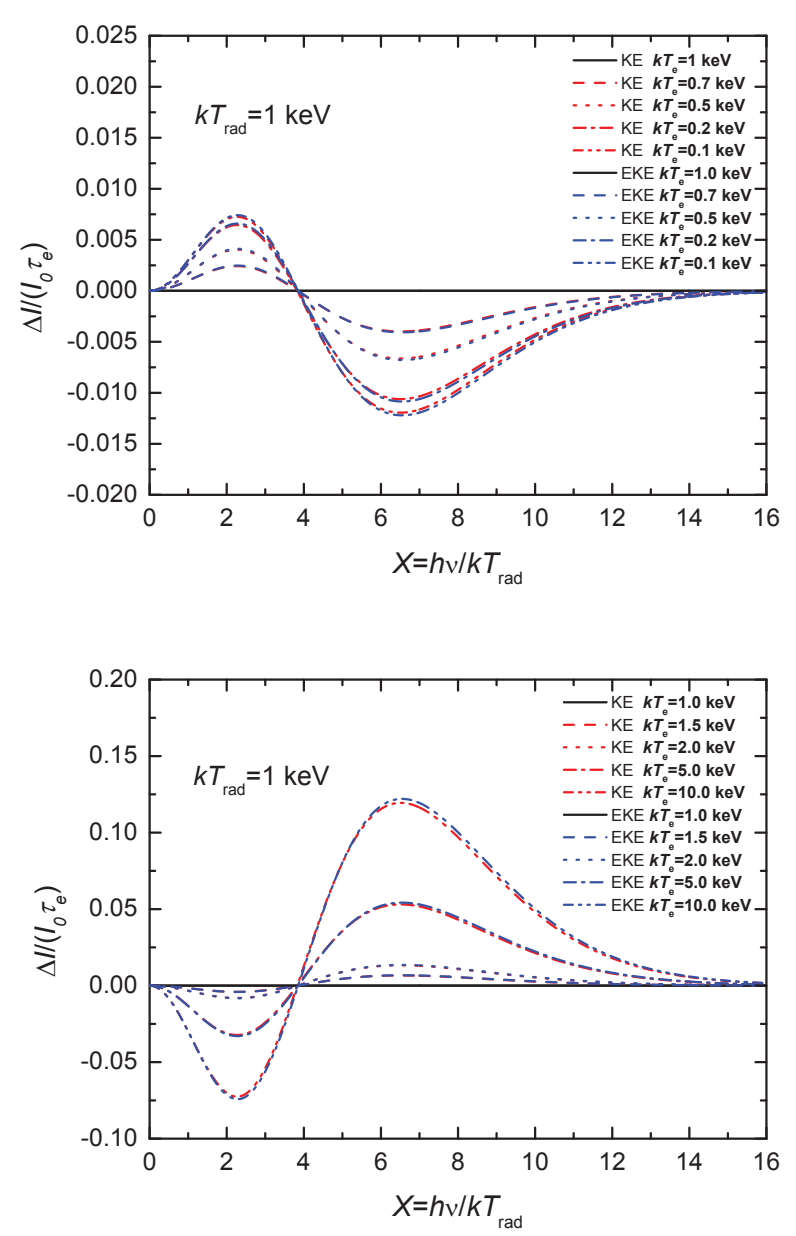

Fig. A.7. Change in intensity of the blackbody spectrum with $k T_{\mathrm{rad}}=$ $1 \mathrm{keV}$ under different temperatures of thermal electron gas calculated by use of Eqs. (39) and (40) as a function of dimensionless frequency $X=h v /\left(k T_{\text {rad }}\right)$, with scaling $I_{0} y_{\mathrm{c}}=\frac{2 h}{c^{2}}\left(\frac{k T_{\text {rad }}}{h}\right)^{3} y_{\mathrm{c}} \cdot k T_{\text {rad }}$ and $k T_{\mathrm{e}}$ are labeled in the figures. The results obtained under the extended Kompaneets equation and the classical Kompaneets equation are shown as solid and red lines, respectively. 

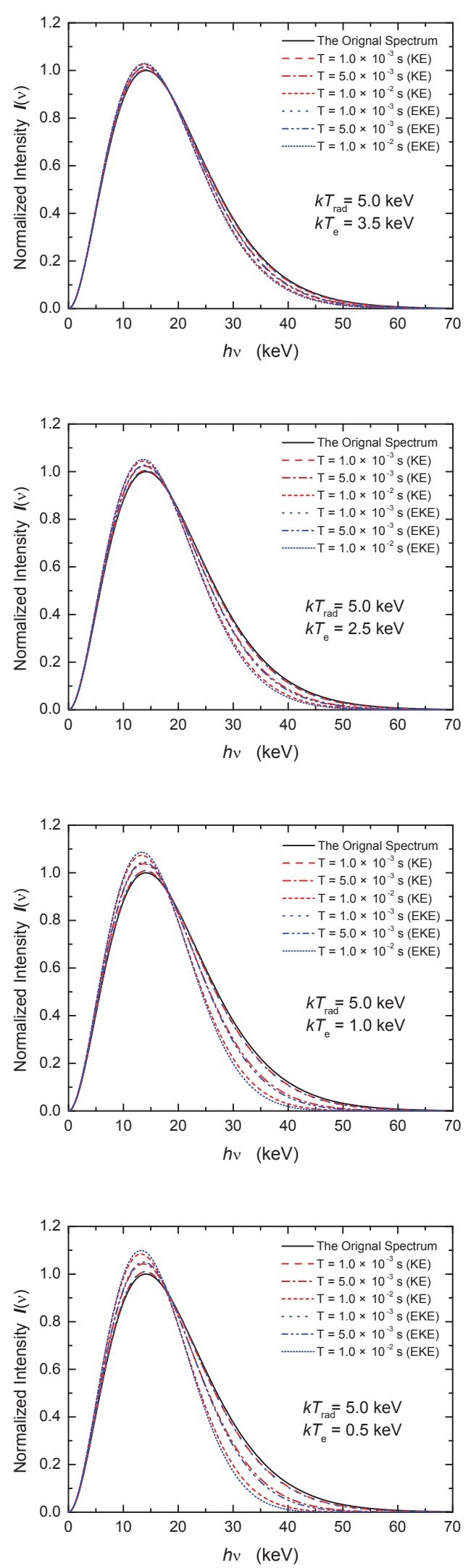

Fig. A.8. Comparison of evolution behavior for the downComptonization of X-ray blackbody spectrum with $k T_{\text {rad }}=5 \mathrm{keV}$ between the classical Kompaneets equation and our extended equation. The evolution times $T, k T_{\text {rad }}$, and $k T_{\mathrm{e}}$ are labeled in the figures. The blue and red lines represent the calculated results of the extended equation and the Kompaneets equation, respectively.
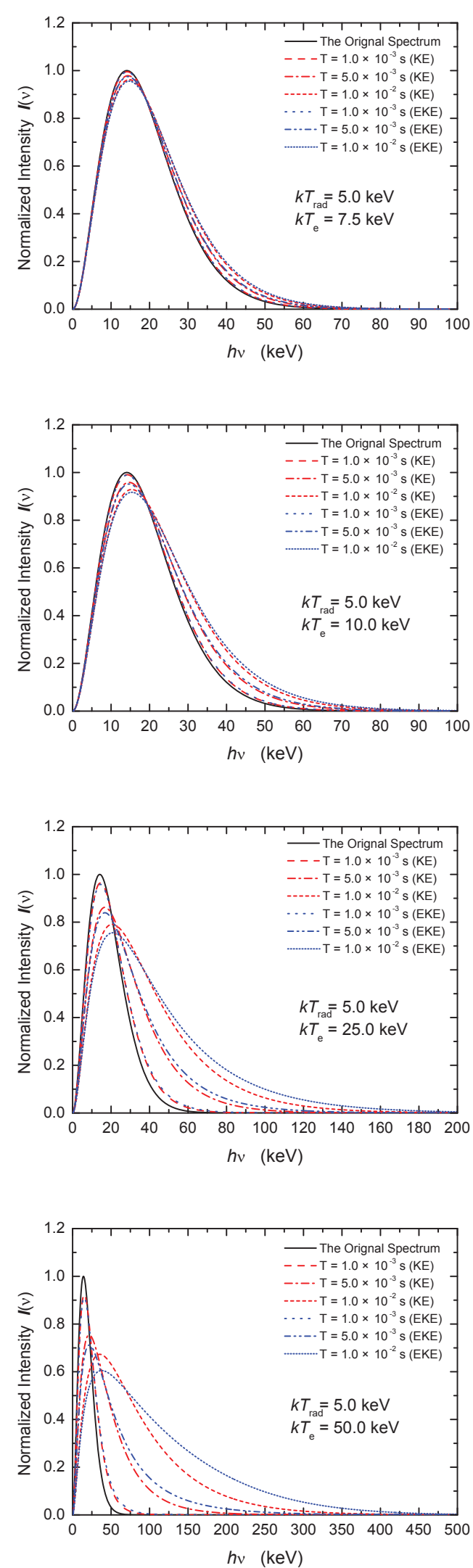

Fig. A.9. Comparison of evolution behavior for the downComptonization of X-ray blackbody spectrum with $k T_{\text {rad }}=5 \mathrm{keV}$ between the classical Kompaneets equation and our extended equation. The evolution times $T, k T_{\text {rad }}$, and $k T_{\mathrm{e}}$ are labeled in the figures. The blue and red lines represent the calculated results of the extended equation and the Kompaneets equation, respectively. 
X. Chen et al.: Modified form of the Kompaneets equation
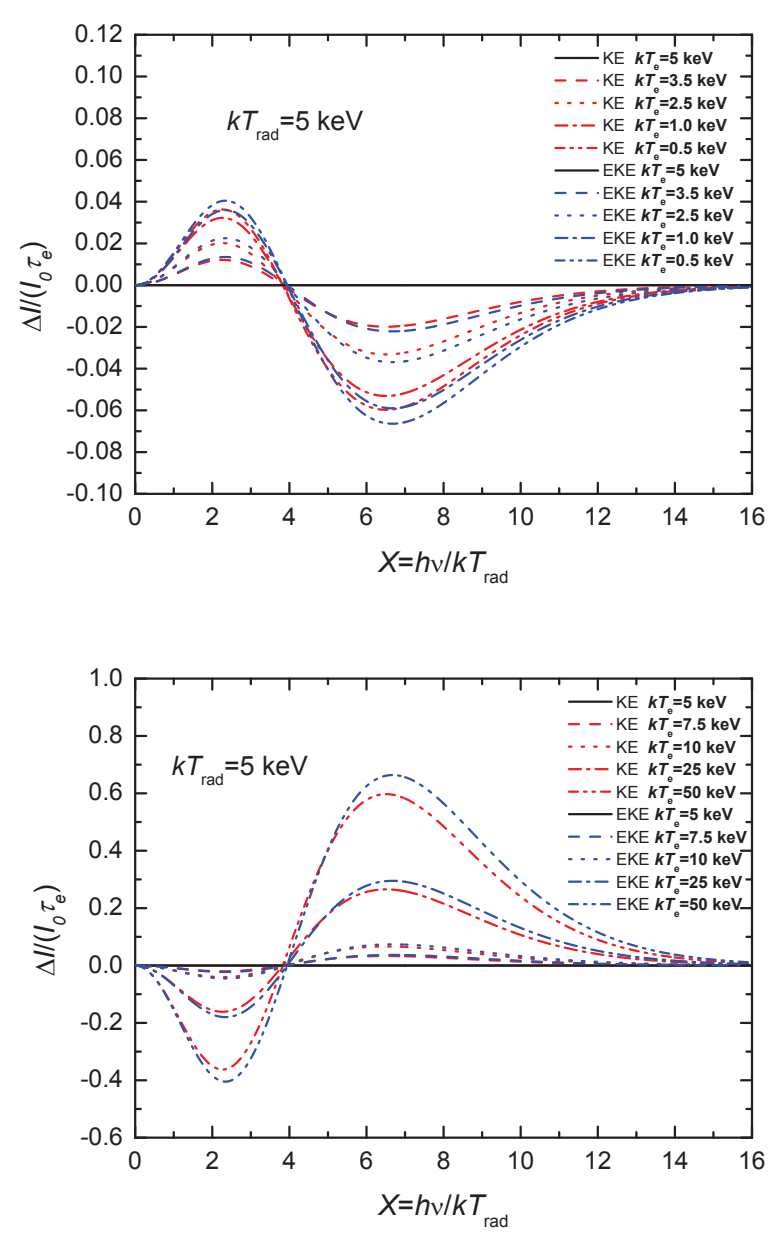

Fig. A.10. Change in intensity of the blackbody spectrum with $k T_{\text {rad }}=$ $5 \mathrm{keV}$ under different temperatures of thermal electron gas calculated by use of Eqs. (39) and (40) as a function of dimensionless frequency $X=h v /\left(k T_{\text {rad }}\right)$, with scaling $I_{0} y_{\mathrm{c}}=\frac{2 h}{c^{2}}\left(\frac{k T_{\mathrm{rad}}}{h}\right)^{3} y_{\mathrm{c}} \cdot k T_{\mathrm{rad}}$ and $k T_{\mathrm{e}}$ are labeled in the figures. The results obtained under the extended Kompaneets equation and the classical Kompaneets equation are shown as solid and red lines, respectively.
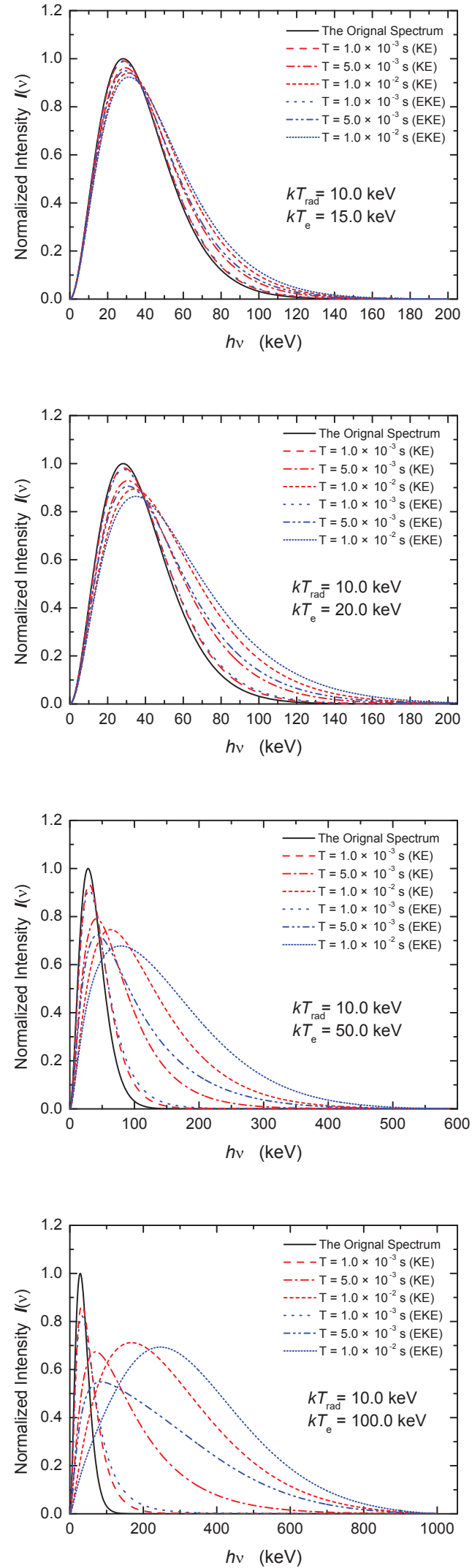

Fig. A.11. Comparison of evolution behavior for the upComptonization of X-ray blackbody spectrum with $k T_{\text {rad }}=10 \mathrm{keV}$ between the classical Kompaneets equation and our extended equation. The evolution times $T, k T_{\text {rad }}$, and $k T_{\mathrm{e}}$ are labeled in the figures. The blue and red lines represent the calculated results of the extended equation and the Kompaneets equation, respectively. 